\title{
Retinal-chitosan Conjugates Effectively Deliver Active Chromophores to Retinal Photoreceptor Cells in Blind Mice and Dogs ${ }^{\mathrm{s}}$
}

\author{
Songqi Gao, ${ }^{1}$ Shirin Kahremany, ${ }^{1}$ Jianye Zhang, Beata Jastrzebska, Janice Querubin, \\ Simon M. Petersen-Jones, and Krzysztof Palczewski
}

\begin{abstract}
Department of Pharmacology and Cleveland Center for Membrane and Structural Biology, School of Medicine, Case Western Reserve University, Cleveland, Ohio (S.G., S.K., J.Z., B.J., K.P.) and Department of Small Animal Clinical Sciences, College of Veterinary Medicine, Michigan State University, East Lansing, Michigan (J.Q., S.M.P.-J.)
\end{abstract}

Received November 28, 2017; accepted February 13, 2018

\section{ABSTRACT}

The retinoid (visual) cycle consists of a series of biochemical reactions needed to regenerate the visual chromophore 11-cis-retinal and sustain vision. Genetic or environmental factors affecting chromophore production can lead to blindness. Using animal models that mimic human retinal diseases, we previously demonstrated that mechanism-based pharmacological interventions can maintain vision in otherwise incurable genetic diseases of the retina. Here, we report that after 9-cis-retinal administration to lecithin:retinol acyltransferase-deficient $\left(\mathrm{Lrat}^{-/-}\right)$mice, the drug was rapidly absorbed and then cleared within 1 to 2 hours. However, when conjugated to form chitosan-9-cis-retinal, this prodrug was slowly absorbed from the gastrointestinal tract, resulting in sustainable plasma levels of 9-cis-retinol and recovery of visual function without causing elevated levels, as occurs with unconjugated drug treatment. Administration of chitosan-9-cis-retinal conjugate intravitreally in retinal pigment epithelium-specific 65 retinoid isomerase (RPE65)-deficient dogs improved photoreceptor function as assessed by electroretinography. Functional rescue was dose dependent and maintained for several weeks. Dosing via the gastrointestinal tract in canines was found ineffective, most likely due to peculiarities of vitamin A blood transport in canines. Use of the chitosan conjugate in combination with 11-cis-6-ring-retinal, a locked ring analog of 11-cisretinal that selectively blocks rod opsin consumption of chromophore while largely sparing cone opsins, was found to prolong cone vision in Lrat $^{-1-}$ mice. Development of such combination low-dose regimens to selectively prolong useful cone vision could not only expand retinal disease treatments to include Leber congenital amaurosis but also the agerelated decline in human dark adaptation from progressive retinoid cycle deficiency.

\section{Introduction}

Production of the active chromophore, 11-cis-retinal (11-cisRAL) is essential for sustaining vision in all mammalian species (von Lintig et al., 2010). This process, termed the retinoid (visual) cycle, takes place in photoreceptor cells and

The authors declare that they have no conflicts of interest with the contents of this article. K.P. is inventor of U.S. Patents No. 8,962,691 and 9,162,978 through the University of Washington whose value may be affected by this publication. Case Western Reserve University may commercialize some of the technology described in this work. K.P. is the Chief Scientific Officer of Polgenix, Inc.

This research was supported in part by grants from the National Institutes of Health (EY009339, EY020551, EY027283, and EY024864 to K.P ; EY025214 to B.J.; EY024864 to K.P.; and core grants P30EY011373 and P30EY025585), and the Myers Dunlap Endowment for Canine Health to S.M.P.-J. K.P. is the John H. Hord Professor of Pharmacology.

${ }^{1}$ S.G. and S.K. contributed equally to this work.

https://doi.org/10.1124/mol.117.111294.

S This article has supplemental material available at molpharm. aspetjournals.org. the retinal pigment epithelium (RPE), a tightly connected twocell system (Kiser et al., 2012, 2014). An additional cycle involving cone pigments and Müller cells has also been postulated (Mata et al., 2002; Wang and Kefalov, 2009). The enzymology of the retinoid cycle has been elucidated in detail at genetic biochemical and structural levels (Lamb and Pugh, 2004; Kiser and Palczewski, 2016). Alterations in genes encoding retinoid-binding proteins and enzymes can result in blinding diseases of varied severity depending on whether an alternative pathway can fulfill a required function (Travis et al., 2007; Kiser and Palczewski, 2016). For example, defects in 11-cis-RAL dehydrogenase usually cause rather mild disease, whereas lack of either lecithin:retinol acyltransferase (LRAT) or retinal pigment epithelium-specific 65 retinoid isomerase (RPE65) lead to the severe early onset blindness Leber congenital amaurosis (LCA) (Travis et al., 2007; Kiser and Palczewski, 2016). Thus, a major effort has been made to devise experimental approaches that alleviate lack of

ABBREVIATIONS: BSA, bovine serum albumin; BTP, Bis-tris propane; CM-chitosan, carboxymethyl-chitosan; DDM, dodecyl- $\beta$-D-maltoside; ERG, electroretinography; GI, gastrointestinal; HPLC, high-performance liquid chromatography; LCA, Leber congenital amaurosis; LRAT, lecithin:retinol acyltransferase; MES, 2-( $N$-morpholino)ethanesulfonic acid; MW, molecular weight; ONL, outer nuclear layer; PBS, phosphate-buffered saline; RAL, retinal; Rho, rhodopsin; ROS, rod outer segment(s); RPE, retinal pigment epithelium; RPE65, retinal pigment epithelium-specific 65-kDa protein or retinoid isomerase; SD-OCT, spectral domain-optical coherence tomography; UV, ultraviolet; Vis, visible. 
chromophore production, including pharmacological (Van Hooser et al., 2000; Batten et al., 2005; Koenekoop et al., 2014; Scholl et al., 2015) and genetic (Bainbridge et al., 2008; Cideciyan et al., 2008, 2013; Maguire et al., 2009) interventions. These initial attempts to correct retinoid cycle deficiencies require further improvement.

We previously demonstrated that mechanism-based pharmacological interventions can maintain and even restore vision in otherwise incurable genetic diseases. Rapid improvement of visual function was observed in patients with RPE65 or LRAT mutations after oral administration of synthetic 9-cis-retinyl acetate (Van Hooser et al., 2000; Batten et al., 2005). Pharmacological doses of 9-cis-RAL also dramatically restored visual function in animal models (mice and dogs) that lack efficient regeneration of 11-cis-RAL due to dysfunctional or suppressed RPE65 and/or LRAT (Van Hooser et al., 2000, 2002; Batten et al., 2004; Maeda et al., 2009a; Gearhart et al., 2010). Systemic pharmacological intervention with activating 9-cis-retinoids, a substitute for much less stable 11-cis-retinoids, has now moved from preclinical studies to clinical trials in patients suffering from LCA caused by inactivating mutations in either LRAT or RPE65 (Koenekoop et al., 2014; Scholl et al., 2015). Moreover, cisretinoid treatment prevented deteriorating vision in aging mice (Maeda et al., 2006), raising hope for a similar outcome in humans.

Two further stages of drug development should be considered to further improve this experimental treatment. First, retinoid drugs should be designed so they are retained safely after unconjugated drug administration to lower their potentially toxic systemic exposure during prolonged treatment. Second, oral delivery of retinoids for treatment of ocular disease is a specialized process involving their gastrointestinal (GI) absorption, carrier-mediated transport in the blood, and specific transfer into the eye through the blood-retina barrier (Kiser et al., 2014). An additional factor for consideration is that artificial illumination in modern life renders rod vision in humans less critical than cone-mediated color vision. So, could a therapy be devised that delivers retinoids in a more sustainable way preferentially to cone photoreceptor cells? To safely prolong drug exposure, possibilities include slowrelease formulations delivered either systemically or by intraocular injection. To help preserve cone vision, rod cells could be selectively blocked with a synthetic retinoid to avoid their consumption of 11-cis-RAL while simultaneously providing a photoactive compound more specifically to cone cells. This is relevant because rhodopsin (Rho) in rods is present in much higher quantities than cone visual pigments in the retina.

Here, we found that after oral administration of unconjugated 9-cis-RAL, the drug was rapidly absorbed, resulting in high circulating levels, and then cleared within 1 to 2 hours, as published previously (Maeda et al., 2009b, 2013). But when conjugated to chitosan, a linear polysaccharide frequently used as a carrier in medical applications (Upadhyaya et al., 2014), to form chitosan-9-cis-RAL, the pro-drug was slowly absorbed from the GI tract, resulting in a continuous sustainable level of 9-cis-RAL without attaining the high levels that occurred with unconjugated drug treatment. In the RPE65deficient dog model, intravitreal delivery of chitosan-9-cisRAL resulted in improvement of both rod and cone function as assessed by electroretinography. By delivering 9-cis-RAL to cone opsin via chitosan conjugation in combination with a locked 11-cis-6-ring analog of RAL to block rod opsin, cone vision was found to be prolonged in mouse and dog models of retinal degeneration.

\section{Materials and Methods}

Animals. Male and female C57BL/6J $\mathrm{Lrat}^{-1-}$ mice at 4-8 weeks of age were used in all experiments. Mice were housed and maintained in a 12-hour light ( $\leq 10$ lux $) / 12$-hour dark cyclic environment or in a dark room in the Animal Resource Center at the School of Medicine, Case Western Reserve University. All animal handling and protocols were approved by the Institutional Animal Care and Use Committee at Case Western Reserve University and conformed to recommendations of both the American Veterinary Medical Association Panel on Euthanasia and the Association for Research in Vision and Ophthalmology.

RPE65-deficient dogs were maintained as a colony at Michigan State University. All procedures adhered to the Association for Research in Vision and Ophthalmology guidelines for the use of animals in ophthalmic and vision research and were approved by the institutional animal care and use committee.

Materials. Carboxymethyl-chitosan (CM-chitosan) was purchased from Santa Cruz Biotechnology (Dallas, TX). All other chemicals were purchased from Sigma Aldrich (St. Louis, MO).

Synthesis of 9-cis-Retinyl Acetate. All-trans-retinyl acetate $(400 \mathrm{~g})$ was dissolved in a solution of hexanes $(800 \mathrm{ml})$ and trimethylamine $(0.22 \mathrm{ml})$. After addition of $0.4 \mathrm{mg}$ of bis(benzonitrile)palladium chloride, the mixture was stirred overnight in the dark at $65^{\circ} \mathrm{C}$ under nitrogen (Supplemental Fig. 1). The resulting solution was cooled to room temperature for 1 hour and then further cooled to $-80^{\circ} \mathrm{C}$. The all-trans-retinyl acetate that crystallized was filtered by suction using a filter precooled with dry ice. The filtrate was concentrated and further crystallized. This process was repeated to obtain a 70:30 mixture of 9-cis-/all-trans-retinyl acetate, which was used for the next steps without further purification.

Synthesis of 3,7-Dimethyl-9-(2,6,6-trimethylcyclohexen-1yl)nona-2,4,6,8-tetraen-1-ol (9-cis-retinol). To a solution of retinyl acetate $\mathbf{1}(\sim 55 \mathrm{ml})$ in ethanol $(181 \mathrm{ml})$, a solution of $\mathrm{NaOH}(20 \mathrm{~g})$ in water $(84 \mathrm{ml})$ was added dropwise at $40^{\circ} \mathrm{C}$ under nitrogen in the dark. After stirring for 30 minutes, the mixture was cooled to $0^{\circ} \mathrm{C}$ and then extracted with hexanes $(3 \times 200 \mathrm{ml})$. The combined organic layers were washed with ice water twice, dried over anhydrous $\mathrm{MgSO}_{4}$, filtrated, and the filtrate was concentrated under vacuum. The resultant yellow oil was dissolved in a solution of pyridine $(0.198 \mathrm{ml})$ in methyl formate $(99 \mathrm{ml})$, stirred at $0^{\circ} \mathrm{C}$ for 2 hours, and then cooled to $-20^{\circ} \mathrm{C}$ for overnight crystallization to obtain compound 2 (Supplemental Fig. 1).

Synthesis of 3,7-Dimethyl-9-(2,6,6-trimethylcyclohexen-1yl)nona-2,4,6,8-tetraenal (9-cis-retinal). To a solution of $2(\sim 30$ g) in dry methylene chloride (300 ml), manganese dioxide (180 g) was added in the dark and the mixture was stirred overnight at room temperature. The mixture was then filtered, and the filtrate was evaporated; both steps were conducted under vacuum. The resulting oil was dissolved in hexanes $(90 \mathrm{ml})$ and cooled to $-80^{\circ} \mathrm{C}$. Crystals from the oil were filtered by suction using a filter precooled with dry ice. ${ }^{1} \mathrm{H} \mathrm{NMR}\left(400 \mathrm{MHz}, \mathrm{CDCl}_{3}\right) \delta 10.09\left(\mathrm{~d}, J=8.2 \mathrm{~Hz},{ }^{1} \mathrm{H}\right), 7.22(\mathrm{dd}, J=$ $\left.15,11.6 \mathrm{~Hz},{ }^{1} \mathrm{H}\right), 6.66\left(\mathrm{~d}, J=15.8 \mathrm{~Hz},{ }^{1} \mathrm{H}\right), 6.33\left(\mathrm{~d}, J=15.8 \mathrm{~Hz},{ }^{1} \mathrm{H}\right)$, $6.30\left(\mathrm{~d}, J=15 \mathrm{~Hz},{ }^{1} \mathrm{H}\right), 6.09\left(\mathrm{~d}, J=11.6 \mathrm{~Hz},{ }^{1} \mathrm{H}\right), 5.97(\mathrm{~d}, J=8.2 \mathrm{~Hz}$, $\left.{ }^{1} \mathrm{H}\right), 2.31\left(\mathrm{~s},{ }^{3} \mathrm{H}\right), 2.05\left(\mathrm{~m},{ }^{2} \mathrm{H}\right), 2.02\left(\mathrm{~s},{ }^{3} \mathrm{H}\right), 1.75\left(\mathrm{~s},{ }^{3} \mathrm{H}\right), 1.64\left(\mathrm{~m},{ }^{2} \mathrm{H}\right), 1.48$ $\left(\mathrm{m},{ }^{2} \mathrm{H}\right), 1.04(\mathrm{~s}, 6 \mathrm{H}) ;{ }^{13} \mathrm{C} \mathrm{NMR}\left(100 \mathrm{MHz}, \mathrm{CDCl}_{3}\right) \delta 191.4,155.2,140.4$, 138.2 , 134.0, 131.5, 131.3, 130.6, 129.5, 129.2, 128.1, 39.64, 34.45 33.28, 29.20, 22.09, 21.21, 19.38, 13.40 (see Supplemental Fig. 1).

11-cis-6-Ring-RAL Synthesis. A mixture of (E)-2-((Z)-4-((E)-4(2,6,6-trimethylcyclohex-1-en-1-yl)but-3-en-2-ylidene)cyclohex-2en-1-ylidene)acetaldehyde (9,11-dicis), (Z)-2-((E)-4-((E)-4-(2,6,6-trimethylcyclohex-1-en-1-yl)but-3-en-2-ylidene)cyclohex-2-en-1-ylidene) 
acetaldehyde (11,13-dicis), (E)-2-((E)-4-((E)-4-(2,6,6-trimethylcyclohex1-en-1-yl)but-3-en-2-ylidene)cyclohex-2-en-1-ylidene)acetaldehyde (11-cis), and (Z)-2-((Z)-4-((E)-4-(2,6,6-trimethylcyclohex-1-en-1-yl) but-3-en-2-ylidene)cyclohex-2-en-1-ylidene)acetaldehyde $(9,11,13$ tricis) was prepared by the method reported by Bhattacharya et al. (1992). Characterization of these compounds was reported previously (Alexander et al., 2017; Gulati et al., 2017).

Synthesis and Characterization of 9-cis-RAL and 11-cis-6-RingRAL Chitosan Conjugates. For synthesis of 9-cis-RAL-chitosan, CM-chitosan, $200 \mathrm{mg}$ in $9 \mathrm{ml}$ of $2 \% 2$-( $N$-morpholino)ethanesulfonic acid (MES), pH 6.3, was mixed with $13 \mathrm{mg}$ or up to $87 \mathrm{mg}$ of 9-cis-RAL in $7 \mathrm{ml}$ ethanol. The mixture was stirred at room temperature for 24 hours in a dark room and then precipitated with ethanol. The color of this mixture changed from light yellow to red. The precipitate was dried in a Speed-Vac. 9-cis-RAL in the supernatant was measured with a UV-visible (UV-Vis) spectrophotometer (Cary 50; Varian, Palo Alto, CA). 9-cis RAL content in the conjugate was determined by subtracting the content of 9-cis-RAL in the supernatant from the original loading amount using the extinction efficient $\varepsilon=$ $36,068 \mathrm{M}^{-1} \cdot \mathrm{cm}^{-1}$ at $373 \mathrm{~nm}$ (Robeson et al., 1955). Molecular weights (MWs) of the CM-chitosan conjugates were estimated by gel filtration chromatography. Samples were loaded onto a Superdex 200 gel filtration column (GE Healthcare, Little Chalfont, UK) equilibrated and eluted with PBS (137 mM NaCl, $2.7 \mathrm{mM} \mathrm{KCl}, 4.3 \mathrm{mM} \mathrm{Na} 2 \mathrm{HPO}_{4}, 1.4 \mathrm{mM}$ $\mathrm{KH}_{2} \mathrm{PO}_{4}$ ) buffer, $\mathrm{pH} 7.0$, at flow rate of $0.5 \mathrm{ml} / \mathrm{min}$.

To synthesize the 11-cis-6-ring-RAL conjugate, CM-chitosan, $245 \mathrm{mg}$ in $10 \mathrm{ml} 2 \% \mathrm{MES}$, pH 6.3, was mixed with $10 \mathrm{mg}$ of 11-cis-6ring-RAL in $7 \mathrm{ml}$ ethanol. The mixture was then processed and its content calculated as described above for 9-cis-chitosan, except that the extinction coefficient used for 11-cis-6-ring-RAL upon gel filtration chromatography was $\varepsilon=24,935 \mathrm{M}^{-1} \mathrm{~cm}^{-1}$ at $376.5 \mathrm{~nm}$ (Brown and Wald, 1956).

Synthesis and Characterization of 9-cis-RAL Chitosan Conjugate (a Large Scale). To a solution of $13.2 \mathrm{~g}$ CM-chitosan in $660 \mathrm{ml}$ of $0.25 \times$ PBS buffer and $400 \mathrm{ml}$ of ethanol, a solution of $1 \mathrm{~g}$ 9-cis-RAL in $62 \mathrm{ml}$ ethanol was added dropwise in the dark. The mixture was stirred at room temperature for 24 hours and then concentrated under vacuum. The resultant was dried by lyophilization to obtain a yellow powder. The concentration of 9-cis-RAL in the conjugate was calculated as described above.

Retinoid Administration. All retinoids were handled under dim red light. 9-cis-RAL or 11-cis-6-ring-RAL was dissolved in ethanol and mixed with soybean oil to achieve a final concentration of $10 \%$ ethanol, except when 9-cis-RAL was dissolved in ethanol prior to oral gavage for drug-in-plasma profile experiments. Chitosan conjugates were ground into small particles $(<120$ mesh) that were suspended in water. Both the soybean oil mixtures and conjugate water suspensions were administrated to $\mathrm{Lrat}^{-1-}$ mice by oral gavage with a 20-G feeding needle.

Retinoid Analysis and Pigment Measurements. Retinoids in the eyes and plasma were extracted under dim red light using previously published methods (Palczewski et al., 1999). Briefly, samples were homogenized in a buffer containing $50 \mathrm{mM}$ 4-morpholinepropanesulfonic acid, $10 \mathrm{mM}$ methoxylamine, $\mathrm{pH} \mathrm{7.0,}$ and $50 \%$ ethanol, extracted by hexanes, dried in a Speed-Vac, and dissolved in acetonitrile before injection into an HPLC column as previously described (Batten et al., 2005). Extracted retinoids dissolved in $75 \%$ acetonitrile were separated on a C18 reverse-phase HPLC column (Zorbax ODS C18, $5 \mu \mathrm{m}, 4.6 \times 250 \mathrm{~mm}$; Agilent, Santa Clara, CA) with a linear gradient of acetonitrile (75\%-100\%) in water and $0.1 \%$ formic acid at a flow rate of $1 \mathrm{ml} / \mathrm{min}$ for 20 minutes. Elution of retinoids was monitored by the absorbance at $360 \mathrm{~nm}$ at room temperature.

Visual pigment levels in the mouse eyes were determined by extraction of a homogenate of two retinas in $1 \%$ dodecyl- $\beta$-D-maltopyranoside (DDM) in $50 \mathrm{mM}$ sodium phosphate buffer, $\mathrm{pH}$ 7.3. Homogenized samples were shaken at $4^{\circ} \mathrm{C}$ for 2 hours, centrifuged at $40,000 \mathrm{~g}$ for 30 minutes, and the spectrum of the supernatant was measured with a UV-Vis spectrophotometer. Freshly prepared hydroxylamine
(20 mM, pH 7.0) was added to the supernatant prior to determining the spectrum. Difference spectra were obtained from spectral measurements made before and after bleaching with white light for 5 minutes. Concentrations of iso-Rho were calculated based on the extinction coefficient, $\varepsilon_{487 \mathrm{~nm}}=43000 \mathrm{M}^{-1} \mathrm{~cm}^{-1}$ (Spalink et al., 1983).

Preparation of Opsin Membranes. Bovine rod outer segment (ROS) membranes were prepared from fresh retinas under dim red light (Papermaster, 1982). Isolated ROS membranes were washed free of membrane-associated proteins with hypotonic buffer composed of $5 \mathrm{mM}$ Bis-tris propane (BTP) and $1 \mathrm{mM}$ EDTA, $\mathrm{pH} 7.5$, by gentle homogenization and subsequent centrifugation at 25,000 $\mathrm{g}$ for $30 \mathrm{~min}$ utes. This procedure was repeated four times at $4^{\circ} \mathrm{C}$. The final membrane pellet was suspended in $10 \mathrm{mM}$ sodium phosphate, $\mathrm{pH}$ 7.0 , and $50 \mathrm{mM}$ hydroxylamine to a $3 \mathrm{mg} / \mathrm{ml}$ concentration of Rho, placed on ice, and illuminated with a $150 \mathrm{~W}$ bulb for 30 minutes. Membranes were pelleted by centrifugation at $16,000 \mathrm{~g}$ for 5 minutes and then washed four times with $10 \mathrm{mM}$ sodium phosphate, $\mathrm{pH} 7.0$, and $2 \%$ bovine serum albumin (BSA) followed by four washes with $10 \mathrm{mM}$ sodium phosphate, $\mathrm{pH} 7.0$, and two washes with $20 \mathrm{mM}$ BTP, $\mathrm{pH} 7.5$, and $100 \mathrm{mM} \mathrm{NaCl}$ at $4^{\circ} \mathrm{C}$. Rho and opsin concentrations were measured with a UV-Vis spectrophotometer and quantified by using the absorption coefficient $\varepsilon_{500 \mathrm{~nm}}=40,600 \mathrm{M}^{-} 1 \mathrm{~cm}^{-1}$ (Wald and Brown, 1953) and $\varepsilon_{280 \mathrm{~nm}}=81,200 \mathrm{M}^{-} 1 \mathrm{~cm}^{-1}$ (Surya et al., 1995), respectively.

In Vitro 9-cis-RAL Uptake from the 9-cis-RAL Conjugate. Fresh rod opsin membranes were suspended in $0.5 \mathrm{ml}$ of $20 \mathrm{mM}$ BTP, $\mathrm{pH} 7.0$, to achieve $0.4 \mathrm{mg} / \mathrm{ml}$ protein concentration, placed in a $1.5 \mathrm{ml}$ Eppendorff tube, and covered with a 12,000 molecular weight cut-off dialysis membrane before being dialyzed against $5 \mathrm{ml}$ of 9-cis-RAL conjugate solution or against CM-chitosan at $4^{\circ} \mathrm{C}$ in the dark for 16 hours or 5 days, respectively. Next, membranes were solubilized by addition of DDM to a final concentration of $10 \mathrm{mM}$ for 15 minutes at room temperature followed by a 10 -minute centrifugation at $16,000 \mathrm{~g}$ at $4^{\circ} \mathrm{C}$. The supernatant fraction was used to measure the absorbance spectra. The control experiment involved the use of BSA alone at $0.4 \mathrm{mg} / \mathrm{ml}$. To quantify the uptake of 9-cis-RAL from the 9-cis-RAL conjugate by rod opsin and BSA, the absorption spectra were measured at $20^{\circ} \mathrm{C}$ with a UV-Vis spectrophotometer.

Rho Purification by 1D4 Immunoaffinity Chromatography. Eyes collected from $\mathrm{Lrat}^{-1-}$ mice either untreated or treated with 9-cis-RAL or 11-cis-6-ring-RAL were homogenized in $2 \mathrm{ml}$ of $10 \mathrm{mM}$ BTP, $\mathrm{pH} 7.5,150 \mathrm{mM} \mathrm{NaCl}, 1 \mathrm{mM}$ EDTA, and protease inhibitors and centrifuged at $13,000 \mathrm{~g}$ for 20 minutes at $4^{\circ} \mathrm{C}$. The supernatant was

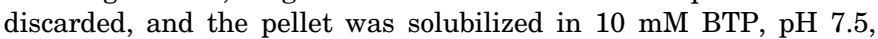
$150 \mathrm{mM} \mathrm{NaCl}$ and $20 \mathrm{mM} \mathrm{DDM}$ for 1 hour at $4^{\circ} \mathrm{C}$. Insoluble material was removed by centrifugation for 1 hour at $100,000 \mathrm{~g}$ and $4^{\circ} \mathrm{C}$. The cleared lysate was used to purify Rho by affinity chromatography on a customized 1D4 antibody-conjugated $\mathrm{CNBr}$ resin (Jastrzebska et al., 2009). The antibody $(6 \mathrm{mg} / \mathrm{ml}$ resin) was coupled to the CNBr-activated Sepharose $4 \mathrm{~B}$ according to the manufacturer's protocol (GE Healthcare Bio-Sciences Corp, Piscataway NJ). Samples were eluted by addition of the 1D4 peptide (TETSQVAPA), and their spectra were subsequently measured with a UV-Vis spectrophotometer.

Electroretinography. ERG responses were recorded as previously described (Maeda et al., 2011). Briefly, dark-adapted mice were anesthetized with a combination of ketamine $(6 \mathrm{mg} / \mathrm{ml})$ and xylazine $(0.44 \mathrm{mg} / \mathrm{ml})$ at a dose of $10 \mu \mathrm{l} / \mathrm{g}$ body weight after their pupils were dilated with $1 \%$ tropicamide. Contact lens electrodes, a reference electrode, and a ground electrode were positioned on the corneas, the head between two ears, and the tail, respectively. For single-flash recording, durations of white-light flash stimuli (from $20 \mu \mathrm{s}$ to $1 \mathrm{~ms}$ ) were adjusted to provide a range of stimulus luminance (from $\sim 3.7$ to $1.6 \mathrm{log} \mathrm{cd} \bullet \mathrm{s} \bullet \mathrm{m}^{-2}$ ). Three to five recordings were made at sufficient intervals between flash stimuli (from 3 seconds to 1 minute) to allow recovery from any photobleaching effects. A UTAS E-3000 (LKC Technologies, Inc., Gaithersburg, MD) was used for ERG recording. 
Spectral Domain-Optical Coherence Tomography Imaging. Ultra-high resolution SD-OCT (Bioptigen, Durham, NC) was used for in vivo imaging of mouse retinas as previously described (Sundermeier et al., 2014). Briefly, mice were anesthetized by intraperitoneal injection of an anesthetic cocktail consisting of ketamine $(6 \mathrm{mg} / \mathrm{ml})$ and xylazine $(0.44 \mathrm{mg} / \mathrm{ml})$ at a dose of $10 \mu \mathrm{l} / \mathrm{g}$ body weight after their pupils were dilated with $1 \%$ tropicamide. Then four frames of OCT images were acquired in the B-scan mode and averaged. Thicknesses of the outer nuclear layers (ONL) were measured in both the superior and inferior retina with the optic nerve head serving as the point of origin.

Histologic Evaluation. Histologic slides were prepared as previously described (Sundermeier et al., 2014). Briefly, mouse eyes were fixed for 24 hours in a solution containing $4 \%$ paraformalaldehyde and $1 \%$ glutaraldehyde in PBS and then processed through a series of ethanol, xylenes, and paraffin in a Tissue-Tek VIP automatic processor according to the manufacturer's instructions (Sakura, Torrance, $\mathrm{CA})$. Sections $(5 \mu \mathrm{m})$ were cut and stained with hematoxylin and eosin (H\&E).

Treatment of RPE65-deficient Dogs. A pilot study showed no effect on ERG recordings after oral dosing with 9-cis-RAL-CMchitosan conjugate. This was anticipated because of the species specific vitamin A metabolism of dogs (Raila et al., 2002). So, for testing in this model, intravitreal administration was performed. 9-cis-RAL-CM-chitosan conjugate [124 mg chitosan containing $3 \mathrm{mg}$ of 9-cis-RAL in $3.5 \mathrm{ml}$ water/oil (50:50), $3 \mathrm{mM}$ final concentration of active compound] was warmed to room temperature and aliquoted in the dark into foil-wrapped syringes with the aid of night vision goggles and injected into the vitreous under dim light conditions. One hundred microliters of full strength conjugate or $100 \mu \mathrm{l}$ of a $50 \%$ or $25 \%$ dilution (dilution series 1, 2, and 4) in balanced salt solution were injected.
Successful injections were performed in two eyes of 8-month-old RPE65-deficient dogs for each concentration (two female dogs were treated unilaterally for $100 \%$ concentration and two males dog bilaterally with $50 \%$ dilution in one eye and $25 \%$ in the contralateral eye). Dogs were maintained initially in the dark for 3 days and then under a very dim light. ERGs were performed as previously described (Annear et al., 2011), except that an Espion $\mathrm{e}^{2}$ visual electrophysiology system with ColorDome Ganzfeld (Diagnosys, Lowell, MA) was used, and only a limited number of stimuli were tested following International Society for Clinical Electrophysiology of Vision protocols (McCulloch et al., 2015).

Statistical Analyses. Results were expressed as the mean \pm S.D. Statistical analyses were performed with Student $t$ test for two-group comparisons, and two-way analysis of variance is used for more than two group comparisons. The individual comparisons were done using Bonferroni's multiple comparisons test. $P$ values $\leq 0.05$ were considered statistically significant.

\section{Results}

The scheme of the synthetic reactions is illustrated in Fig. 1 and Supplemental Fig. 1. 9-cis-RAL-CM-chitosan conjugate (compound B) was synthesized by reacting CM-chitosan (compound a) with 9-cis-RAL in MES buffer, $\mathrm{pH}$ 6.3. The Schiff-base 9-cis-RAL-CM-chitosan conjugate then could either be cleaved by hydroxylamine to produce CM-chitosan (compound D) and 9-cis-RAL oxime or the imine group (compound $\mathrm{C}$ ) in the Schiff base could be reduced by sodium borohydride $\left(\mathrm{NaBH}_{4}\right)$ to form an $\mathrm{N}-\mathrm{C}$ bond that connects CM-chitosan to 9-cis-retinoid in the new conjugate (compound D).

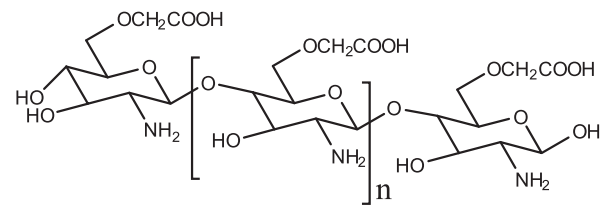

A<smiles>CC1=C(/C=C/C(C)=C\C=C\C(C)=C\C=O)C(C)(C)CCC1</smiles>

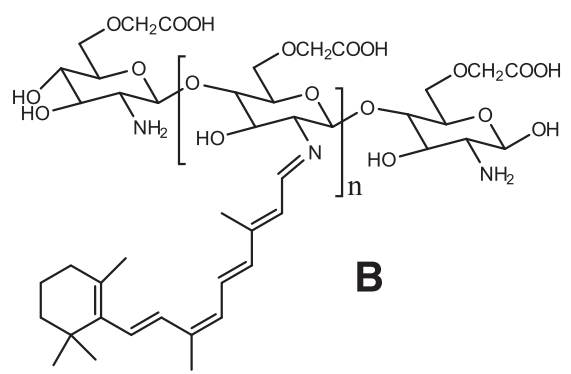
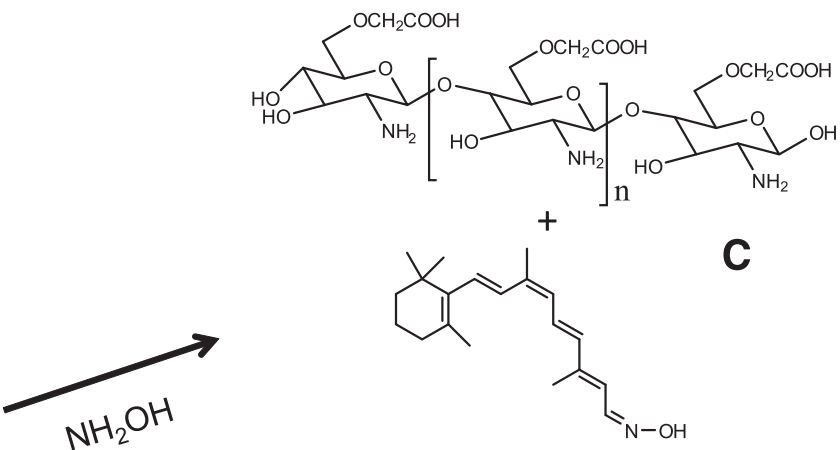

C

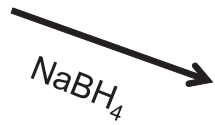

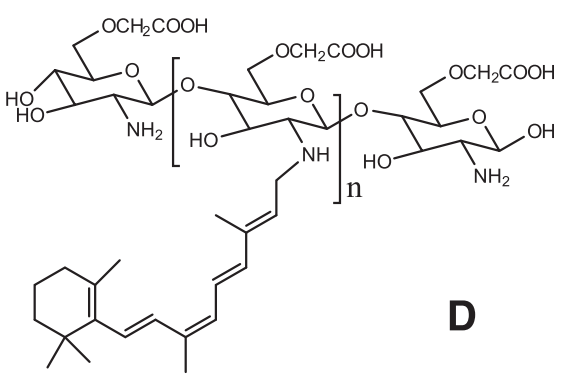

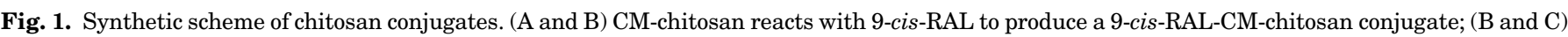

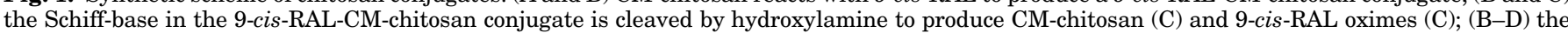

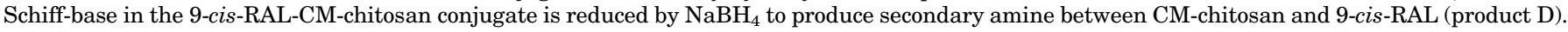


Preincubation of CM-chitosan with $\mathrm{NaBH}_{4}$ did not affect the formation of the 9-cis-RAL CM-chitosan conjugate (compound B) from CM-chitosan and RAL.

The 9-cis-RAL-CM-chitosan conjugate and CM-chitosan were characterized by their UV-Vis spectra, and their MW was estimated by gel filtration chromatography. The UV-Vis spectra and gel filtration profiles are shown in Supplemental Fig. 2, A and B, respectively. The maximum absorbance of the 9-cis-RAL-CM-chitosan conjugate (compound B) was $350 \mathrm{~nm}$. After reduction, the maximum absorbance of the CM-chitosan conjugate (compound e) shifted to the shorter wavelength of $315 \mathrm{~nm}$. There was no absorbance peak between 250 and $600 \mathrm{~nm}$ for CM-chitosan (compounds A, D). Dextran standards were used to estimate the MWs of the 9-cis-RAL-CM-chitosan conjugates. The dextran structure is similar to that of chitosan, and both are polysaccharides. As shown in Supplemental Fig. 2B, the $80 \mathrm{kDa}$ dextran standard peak and the 9-cis-RAL-CM-chitosan conjugate peak were similar. Therefore, the MW of the 9-cis-RAL-CM-chitosan conjugate was estimated at about $80 \mathrm{kDa}$.

Release of 9-cis-RAL from the 9-cis-RAL Conjugate and Its Uptake by Opsin in Vitro. To test the release of
9-cis-RAL from the 9-cis-RAL conjugate and its ability to regenerate iso-Rho, a suspension of opsin rod outer segment (ROS) membranes was dialyzed against the 9-cis-RAL conjugate solution. This allowed only released 9-cis-RAL to penetrate the dialysis membrane and associate with opsin. Regeneration of iso-Rho was determined by measurement of the UV-Vis absorption spectra either after 16 hours or 5 days of dialysis. A small but distinct absorption peak was detected at $485 \mathrm{~nm}$ after 16 hours of incubation (Fig. 2A, red spectrum) that increased after 5 days of dialysis (Fig. 2B, red spectrum). This indicates that both the release of 9-cis-RAL from the 9-cis-RAL conjugated and its uptake by opsin resulted in the regeneration of iso-Rho compared with the sample incubated with CM-chitosan only (Fig. 2, A and B, black spectra). BSA containing a hydrophobic pocket in its structure that can accommodate small hydrophobic molecules was used for the control experiment. The uptake of RAL released from the RAL-conjugate CM-chitosan was determined by UV-Vis spectroscopy as an increase in absorption at $\sim 360 \mathrm{~nm}$. Although only a negligible change was observed after 16 hours (Fig. 2C), a significant spectral change was detected after 5 days (Fig. $2 \mathrm{D}$ ), indicating again the ability of 9-cis-RAL released from the
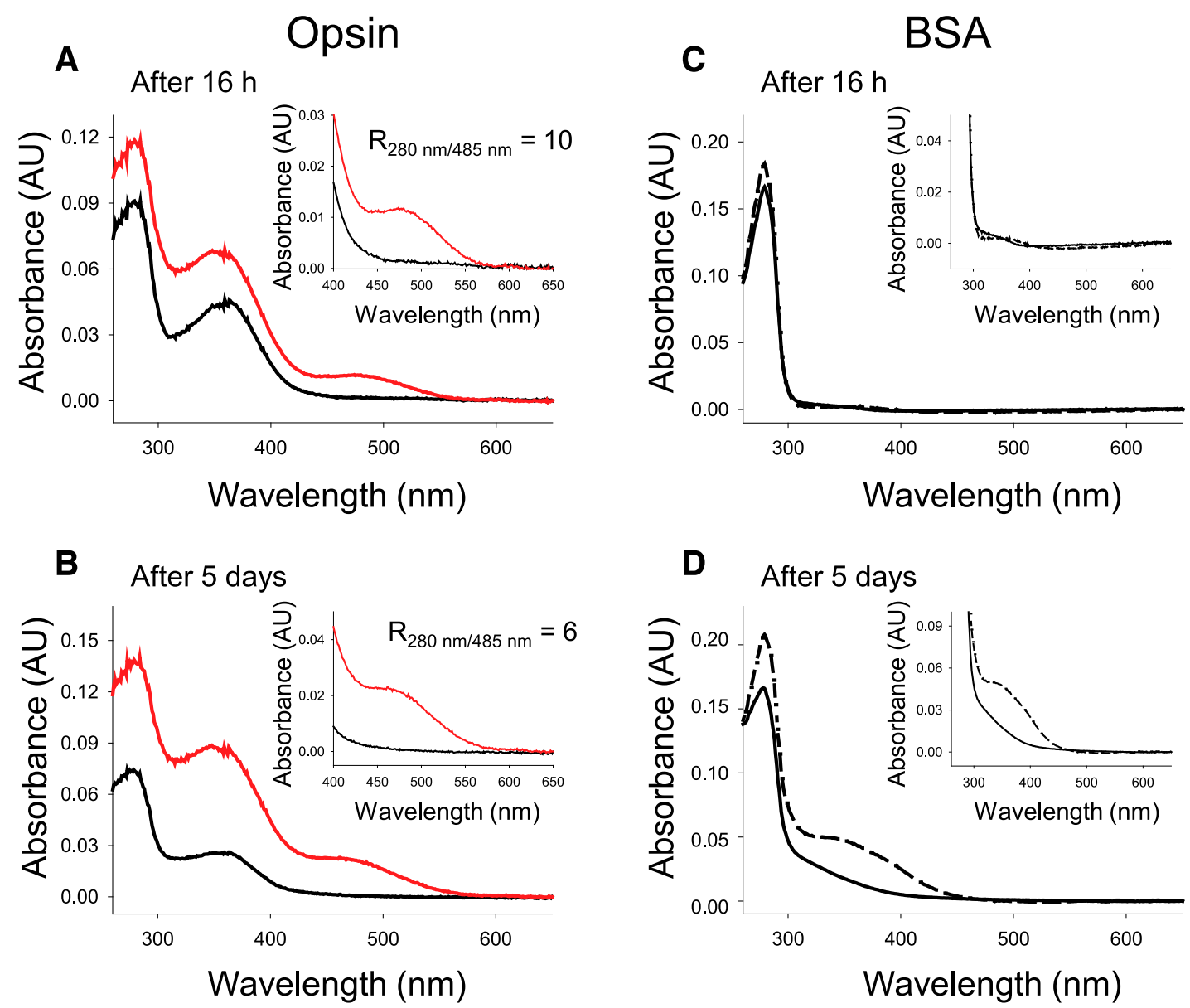

Fig. 2. Uptake of 9-cis-RAL from the 9-cis-RAL conjugate by rod opsin. The uptake of 9-cis-RAL from the 9-cis-RAL conjugate by opsin membranes and BSA was measured. (A and B) UV-Vis absorption spectra measured after 16 hours or 5 days dialysis of opsin membranes against either the 9-cis-RAL conjugate (red spectrum) or CM-chitosan (black spectrum). Detection of an absorption maximum peak at 485 nm indicates the release of 9-cis-RAL from the CM-chitosan conjugate and its uptake by opsin within the indicated time. (C and D) BSA was dialyzed against either the 9-cis-RAL conjugate solution (solid black spectrum) or CM-chitosan solution alone (dashed black spectrum) and UV-Vis absorption was measured after 16 hour and 5 days. The increase in absorbance at about $360 \mathrm{~nm}$ indicates the release of 9-cis-RAL from the CM-chitosan conjugate and its uptake by BSA within the indicated time. Inserts show expended spectra. 


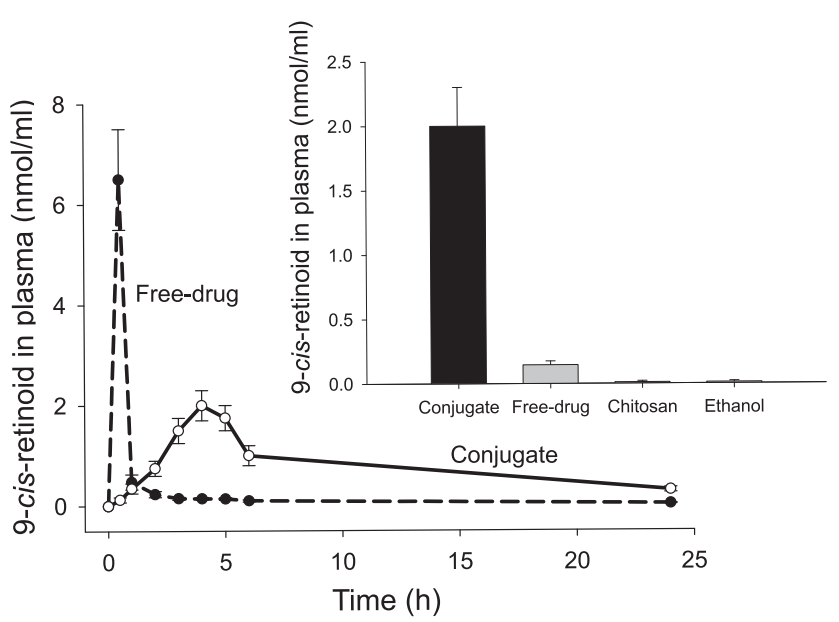

Fig. 3. 9-cis-Retinoid concentrations in the plasma of $\mathrm{Lrat}^{-1-}$ mice after a single dose of free 9-cis-RAL or 9-cis-RAL-CM-chitosan conjugate. The free drug, its conjugate (CM-chitosan), and $10 \%$ ethanol in soybean oil were administrated by gastric gavage at a dose of $50 \mathrm{mg} / \mathrm{kg}$ of the 9 -cis-RAL equivalent. Closed circles represent data for free, and open circles show conjugated 9-cis-RAL. Insert: 9-cis-retinoid concentration in the plasma of $\mathrm{Lrat}^{-1-}$ mice 4 hours after compound administration. Values are expressed as mean \pm S.D., $n=5$ for each time point. Comparing two groups, conjugate to free drug, conjugate to chitosan, conjugate to ethanol, the $P$ values were $<0.001$.

9-cis-RAL conjugate to associate with the hydrophobic binding pocket of certain proteins.

9-cis-Retinoid Levels in Plasma after Treatment. To evaluate the capability of the CM-chitosan conjugates to maintain sustained release of 9-cis-retinoid after oral administration, 9-cis-retinoid levels in plasma were measured by HPLC. $\mathrm{Lrat}^{-1-}$ mice received the CM-chitosan conjugates or free drug by oral gavage at a dose of $50 \mathrm{mg} / \mathrm{kg}$ of either 9-cisRAL conjugate or 9-cis-RAL. Their plasma was collected at scheduled time points and analyzed by HPLC. As shown in Fig. 3, 9-cis-retinoid levels in plasma of the CM-chitosan conjugate-treated mice increased gradually, reaching a peak at about 4 hours after oral gavage and then decreased slowly. Even 24 hours later, 9-cis-retinoid was detected in the plasma of CM-chitosan conjugate-treated mice. In contrast, 9-cisretinoid levels in the plasma of unconjugated drug-treated mice increased rapidly, reaching a peak within 1 hour after oral gavage, and then sharply dropped, being barely detectable 10 hours later. The 9-cis-retinoid peak in plasma of unconjugated drug treated mice was 3.2-fold higher than that of CM-chitosan conjugate-treated mice. This higher drug peak, above the therapeutic window, could potentially cause toxicity, and the rapid drug clearance noted in the unconjugated drug-treated group would certainly result in lower drug bioavailability. No 9-cis-retinoid was detected in mice administered only with chitosan or ethanol in the control experiment.

Eyes of $\mathrm{Lrat}^{-/-}$Mice Accumulated Higher Levels of 9-cis-Retinoid after Oral Administration of the 9-cisRAL Conjugate. HPLC was used to determine 9-cis-retinoid levels in the eyes of $\mathrm{Lrat}^{-1-}$ mice as shown by a representative chromatogram in Supplemental Fig. 3. $\mathrm{Lrat}^{-1-}$ mice were gavaged with the CM-chitosan conjugate or free drug at a dose of $45 \mathrm{mg} / \mathrm{kg}$ of 9-cis-RAL conjugate or 9-cis-RAL 24 hours prior to analysis. Eye samples were collected, homogenized in a buffer containing $50 \mathrm{mM}$ 4-morpholinepropanesulfonic acid and $50 \mathrm{mM} O$-methylhydroxylamine, $\mathrm{pH} 7.0$, extracted, and examined by reverse-phase HPLC. The 9-cis-RAL ( $O$-methyl)oxime peaks from the eye samples were identified based on their elution times and absorbance spectra. The maximum UV absorbance of 9-cis-RAL ( $O$-methyl)oxime was at $358 \mathrm{~nm}$. The chromatogram reveals that the 9-cis-retinoid peak from the eyes of the CM-chitosan conjugate-treated mice was larger than that of unconjugated drug-treated mice, whereas there
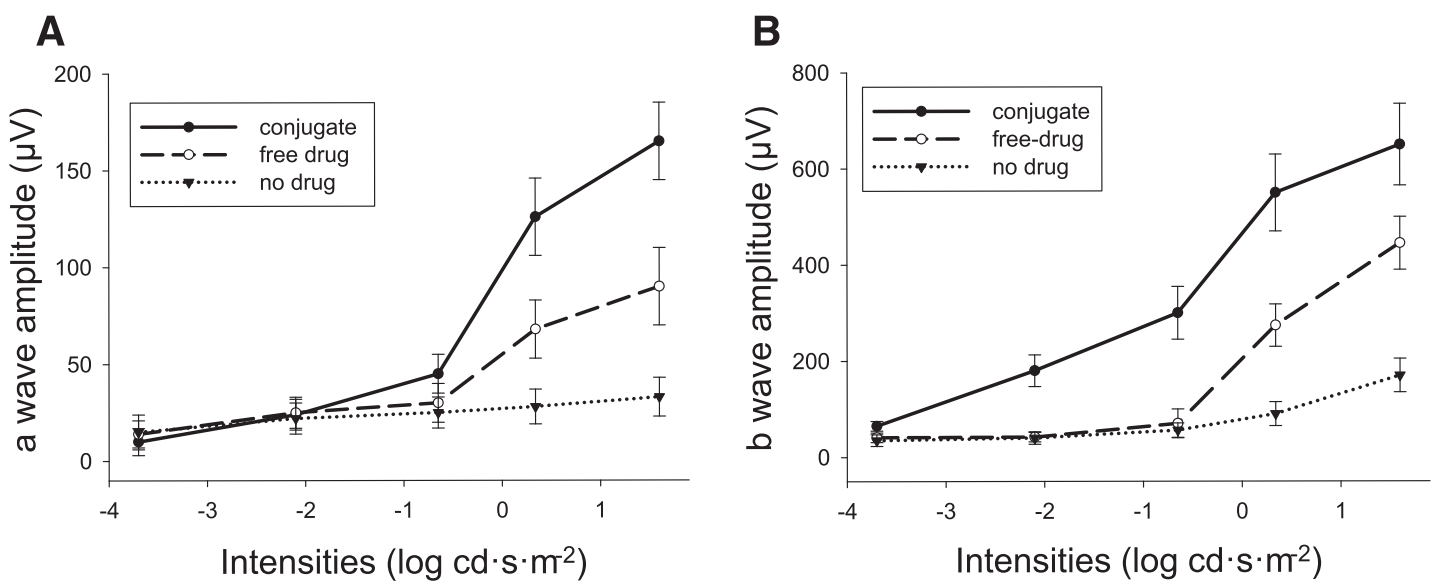

Fig. 4. Improvement of retinal function in $\mathrm{Lrat}^{-1-}$ mice treated with 9-cis-RAL-CM-chitosan conjugate. Mice received the conjugate or free drug by oral gavage at a dose of $35 \mathrm{mg} / \mathrm{kg}$ of 9-cis-RAL or the 9-cis-RAL equivalent, and then were kept in the dark. Scotopic single-flash ERG responses were recorded 3 days after drug administration. a-Wave amplitudes (A) of ERG responses were significantly higher $(P<0.001)$ when stimulating light intensity reached 0.34 and $1.6 \log \mathrm{cd} \cdot \mathrm{s} \cdot \mathrm{m}^{-2}$ in the conjugate treated-group compared with the free drug-treated group. For lower intensity, when comparing conjugate to free drug, data analyses by two-way analysis of variance (ANOVA) yielded the $P$ value $>0.05$ at intensities equal to $-3.7,-2.1$, and -0.65 (log cd.s.m ${ }^{-2}$ ). $P$ value was $<0.01$ at intensities equal to 0.34 and $1.6\left(\log \mathrm{cd} \cdot \mathrm{s} \cdot \mathrm{m}^{-2}\right)$. When comparing conjugate to no drug at intensities equal to $-3.7,-2.1$, and -0.65 (log $\left.\mathrm{cd} \cdot \mathrm{s} \cdot \mathrm{m}^{-2}\right)$, the $P$ value was $>0.05$; and at 0.34 and $1.6\left(\log \mathrm{cd} \cdot \mathrm{s} \cdot \mathrm{m}^{-2}\right)$, the $P$ value was $<0.001$. (B) b-Wave amplitudes of ERG responses were significantly greater $(P<0.001)$ in the conjugate treated-group than in the free drug-treated group. For lower intensity, when comparing conjugate to free drug at intensity equal to $-3.7\left(\log \mathrm{cd} \cdot \mathrm{s} \cdot \mathrm{m}^{-2}\right)$, the $P$ value was $>0.05$, and for intensities equal to: $-2.1,-0.65,0.34$, and $1.6\left(\log \mathrm{cd} \cdot \mathrm{s} \cdot \mathrm{m}{ }^{-2}\right)$, the $P$ values were $<0.01$. When comparing conjugate to no treatment at $-3.7\left(\log \mathrm{cd} \cdot \mathrm{s} \cdot \mathrm{m}^{-2}\right)$, the $P$ value was $>0.05$, and at intensities equal to $-2.1,-0.65,0.34$, and 1.6, the $P$ value was $<0.001$. Data analyses were performed by two-way ANOVA. Values are expressed as mean \pm S.D., $n=9$ for each group. 

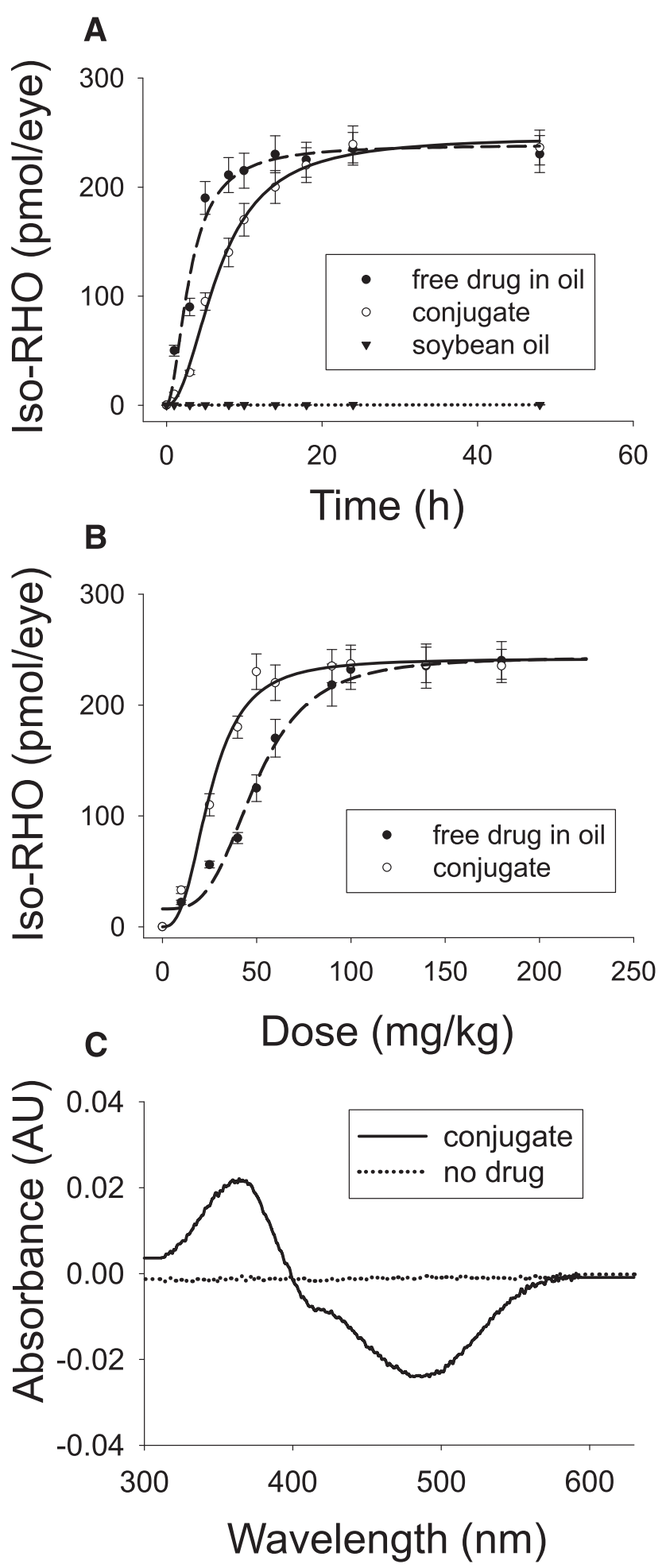

Fig. 5. Iso-Rho levels in the eyes of $\mathrm{Lrat}^{-1-}$ mice after oral administration of 9-cis-RAL-CM-chitosan conjugate or free 9-cis-RAL. (A) isoRho levels vs. time after an oral dose of $150 \mathrm{mg} / \mathrm{kg}$ of free drug or conjugate. The half-time to reach the plateaus is 3.05 hours for free drug and 6.74 hours for conjugate. Comparing values for conjugate to free drug, data analyses by two-way ANOVA at times equal to $1,3,5,8$, and 10 hours yielded $P$ values $<0.01$ and at times equal to $14,18,24$, and 48 hours, $P$ values $>0.05$. (B) iso-Rho levels vs. dosage 48 hours after oral administration of free drug or conjugate. The half-dose to reach the plateaus is $49.6 \mathrm{mg} / \mathrm{kg}$ for free drug and $25.4 \mathrm{mg} / \mathrm{kg}$ for conjugate; the maximum plateau is $241.4 \mathrm{pmol} / \mathrm{eye}$. Comparing values for conjugate to free drug at doses of $10,25,40,50$, and $60 \mathrm{mg} / \mathrm{kg}, P$ values were $<0.01$, and at $90,100,140,180 \mathrm{mg} / \mathrm{kg}, P$ values were $>0.05$. was no 9-cis-retinoid peak from the eyes of untreated $\mathrm{Lrat}^{-1-}$ mice. These higher ocular 9-cis-retinoid levels detected by HPLC indicate that more visual chromophore is delivered to the eyes of CM-chitosan conjugate-treated mice compared with unconjugated drug treated mice.

Improvement of Retinal Function after Treatment with the 9-cis-RAL Conjugate. To test retinal function, we recorded the ERG responses of $\mathrm{Lrat}^{-{ }^{-1}}$ mice treated with the CM-chitosan conjugate or free drug, and the a-wave and b-wave amplitudes of the ERG responses are shown in Fig. 4. Lrat $^{-1-}$ mice were gavaged with either the CM-chitosan conjugate or free drug at a dose of $35 \mathrm{mg} / \mathrm{kg}$ of 9-cis-RAL conjugate or 9-cisRAL and kept in the dark room for 3 days prior to single-flash scotopic ERG recording. Delivery of 9-cis-retinoid from the CM-chitosan conjugate to the eyes caused significantly increased ERG responses as shown in both a-wave and b-wave amplitudes beginning at a stimulus intensity of 0.34 and $-2.08 \log \mathrm{cd} \bullet \mathrm{s}^{\bullet} \cdot \mathrm{m}^{-2}$, respectively, compared with those of unconjugated drug-treated mice. Since both the CM-chitosan conjugate and free drug treatment can deliver visual chromophore to the eyes of $\mathrm{Lrat}^{-1-}$ mice, the ERG responses of both groups were higher than those of the control group. This ERG response pattern suggests greater improvement of retinal function in the CM-chitosan conjugate treated group than in the free drug-treated $\mathrm{Lrat}^{-1-}$ mice.

Restored Visual Pigment iso-Rho in Eyes after Oral Gavage with 9-cis-RAL Conjugate. In some inherited retinal diseases, mutations in retinoid cycle genes such as Lrat cause a decreased supply of the visual chromophore 11-cis-RAL (Batten et al., 2004, 2005). Thus pharmacological replacement of the missing chromophore is an efficient way to treat this type of disease. An artificial chromophore, 9-cisRAL, was selected to replace the missing chromophore because it is easier to synthesize and thermodynamically more stable than 11-cis-RAL. 9-cis-RAL binds with opsin to form iso-Rho, which, when bleached, undergoes conformational changes through the same photoproducts as 11-cisRAL-regenerated Rho. To confirm the formation of endogenous visual pigment, we measured iso-Rho levels at different time points and dosages after oral gavage with either 9-cisRAL or 9-cis-RAL conjugate. The findings are shown in Fig. 5. With CM-chitosan conjugates or free drug given at a dose of $150 \mathrm{mg} / \mathrm{kg}$ of 9 -cis-RAL conjugate or 9-cis-RAL, iso-Rho gradually accumulated in the $\mathrm{Lrat}^{-/-}$mouse retina, achieving a plateau level of $230 \pm 20 \mathrm{pmol} / \mathrm{eye}$ at 14 hours for the free drug group and at 24 hours for the CM-chitosan conjugate group (Fig. 5A). This level corresponds to $\sim 50 \%$ of the level of visual pigment found in age-matched WT mice (400-600 pmol/eye). There was no iso-Rho accumulation in the eyes of $\mathrm{Lrat}^{-/-}$mice that received only soybean oil as a control. The iso-Rho accumulation rate in the CM-chitosan conjugatetreated group was slower than that of the free drug-treated group because 9-cis-RAL had to be first released from the conjugate before its systemic delivery could occur. However, slower but sustained drug release from the longer lasting CM-chitosan conjugates is desired to prevent its toxicity and prolong its effect. In fact, our in vitro experiment shown in Fig. 2

(C) Absorbance spectrum of iso-Rho from eyes of $\mathrm{Lrat}^{-1-}$ mice gavaged with the conjugate or without any drug. Values are expressed as mean \pm S.D., $n=5$ for each group. 
indicated that a longer time is needed to regenerate iso-Rho when opsin membranes are incubated with 9-cis-RAL conjugate compared with free retinoid (McKibbin et al., 2007; Jastrzebska et al., 2013; Tian et al., 2017). To test the dosage effect on pigment accumulation, $\mathrm{Lrat}^{-/-}$mice were gavaged with the CM-chitosan conjugate or free drug at doses ranging from 0 to $180 \mathrm{mg} / \mathrm{kg}$ of 9-cis-RAL conjugate or 9-cis-RAL 2 days prior to analysis. Iso-Rho accumulated gradually in the retinas with increasing drug doses, reaching a plateau level of $235 \pm$ $21 \mathrm{pmol} / \mathrm{eye}$ at $70 \mathrm{mg} / \mathrm{kg}$ for the CM-chitosan conjugatetreated group and $100 \mathrm{mg} / \mathrm{kg}$ for the unconjugated drugtreated group (Fig. 5B). Before reaching the plateau level, more iso-Rho accumulated in the retinas of CM-chitosan conjugatetreated mice than in free drug-treated mice at the same dosage. The presence of iso-Rho was confirmed by UV-Vis spectroscopy, wherein its maximum absorbance wavelength was $487 \mathrm{~nm}$ (Spalink et al., 1983) (Fig. 5C); this is blue-shifted by $12 \mathrm{~nm}$ compared with Rho at $498 \mathrm{~nm}$.

Percentages of 9-cis-retinoid Contained in the CM-Chitosan Conjugates Affect Ocular iso-Rho Levels and ERG Responses. To examine the influence of water solubility of the CM-chitosan conjugates on visual pigment formation and retinal function, we used four CM-chitosan conjugates containing 6\% (water soluble), 10\% (partially water soluble), $20 \%$ (water insoluble), and 30\% (water insoluble) 9-cis-retinoid (w/w). Lrat $^{-/-}$mice were gavaged with the CM-chitosan conjugates at the same dose of $90 \mathrm{mg} / \mathrm{kg}$ of 9-cis-RAL equivalent 2 days prior to analysis of iso-Rho levels and ERG recording. Oral administration of the CM-chitosan conjugates at the same dose of 9-cis-retinoid led to different levels of ocular iso-Rho due to variations in the water solubility of the CM-chitosan conjugates (Supplemental Fig. 4A). A water insoluble conjugate with 30\% 9-cis-retinoid content led to a significantly lower ocular level of iso-Rho, $142 \pm 28 \mathrm{pmol} / \mathrm{eye}$ compared with $236 \pm 29 \mathrm{pmol} / \mathrm{eye}$ for a more water-soluble conjugate (6\% 9-cis-retinoid content, $P<$ 0.001). In addition, ERG responses were recorded to assess the impact of 9-cis-retinoid content in the CM-chitosan conjugates on retinal function. Amplitudes of b-waves at $1.6 \log \mathrm{cd} \cdot \mathrm{s} \cdot \mathrm{m}^{-2}$ under scotopic conditions are shown in Supplemental Fig. 4B, wherein a 9-cis-retinoid content-dependent ERG response was recorded. Here the water-soluble and lower 9-cis-retinoid content $(6 \%)$ conjugate produced significantly higher $b$-wave amplitudes $(700 \pm 110 \mu \mathrm{V})$ compared with the waterinsoluble and higher 9-cis-retinoid content (30\%) conjugate $(390 \pm 80 \mu \mathrm{V})(P<0.001)$. These data also indicate that the pattern of ERG responses is similar to that of ocular iso-Rho accumulation.

Effect of 9-cis-RAL-CM-Chitosan Conjugate Treatment on Retinal Pathology. To assess the effect of 9-cisRAL-CM-chitosan conjugate treatment on retinal morphology, we gavaged 4-week-old $\mathrm{Lrat}^{-1-}$ mice with the CM-chitosan conjugate at a dose of $80 \mathrm{mg} / \mathrm{kg}$ of 9 -cis-RAL equivalent every 3 days for 4 weeks. Figure 6, A and B, displays H\&E-stained paraffin sections from the eyes of 8-week-old $\mathrm{Lrat}^{-1-}$ mice treated with or without the CM-chitosan conjugate. Histologic analysis showed that multiple gavages effectively improved retinal morphology. The ROS of the conjugate-treated mice was thicker and more tightly packed than that of untreated control mice. The thickness of the ROS layer was substantially improved from $9.0 \pm 1.0 \mu \mathrm{m}$ in control mice to $12.3 \pm 1.0 \mu \mathrm{m}$ for the conjugate-treated mice (Fig. 6C). Because ROS structural
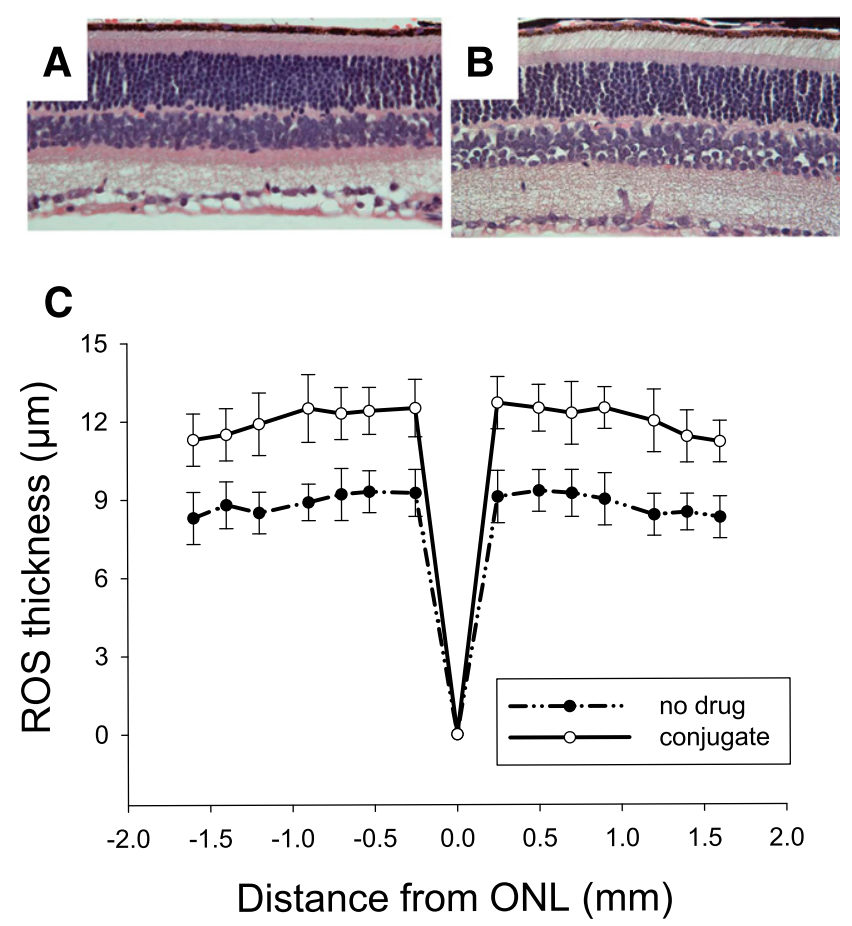

Fig. 6. Improvement of ROS morphology in $\mathrm{Lrat}^{-1-}$ mice treated with 9-cis-RAL-CM-chitosan conjugate. (A and B) H\&E staining of paraffin sections from the eyes of 8-week-old $\mathrm{Lrat}^{-1-}$ mice treated with (A) or without (B) the conjugate for 4 weeks at a dose of $80 \mathrm{mg} / \mathrm{kg}$ of the $9-c i s-\mathrm{RAL}$ equivalent every 3 days. (C) Quantification of ROS thickness in $\mathrm{Lrat}^{-1-}$ mice; the ROS layer in the conjugate-treated group was thicker than in the untreated control group. Data are expressed as mean \pm S.D., $n=9$ for each group. After data analyses by two-way ANOVA at each distance point, the $P$ value was $<0.01$.

morphology depends on functional Rho, regeneration of visual pigments preserves retinal morphology. Furthermore, visual pigment formation requires 9-cis-retinoid that can be supplied efficiently with sustained release of this retinoid from CM-chitosan conjugates. Our UV-Vis spectra showed that opsin in the retina was regenerated and formed iso-Rho (Fig. 5C), suggesting this opsin was properly folded.

We also analyzed retinal morphology in 8-week-old $\mathrm{Lrat}^{- \text {/- }}$ mice using ultra-high-resolution SD-OCT to assess the treatment efficacy of CM-chitosan conjugates. At a younger age, the retinal layers of $\mathrm{Lrat}^{-1-}$ mice are similar to those of WT mice. But at 8 weeks of age, the ONL thickness of $\mathrm{Lrat}^{-1-}$ mice is $45 \pm 3 \mu \mathrm{m}$ compared with $50 \pm 3 \mu \mathrm{m}$ for WT mice. SD-OCT images revealed that the ONL morphology of $\mathrm{Lrat}^{-1-}$ mice treated with 9-cis-RAL-CM-chitosan conjugate appears unchanged compared with untreated mice. Supplemental Fig. 5, A and B, shows that SD-OCT images of retinas from 8-weekold $\mathrm{Lrat}^{-1-}$ mice treated with or without the conjugate. Quantification of ONL thicknesses in $\mathrm{Lrat}^{-/-}$mice demonstrated that there was no significant difference between conjugate-treated and untreated control groups (Supplemental Fig. 5C).

The 11-cis-6-ring-RAL-CM-chitosan Conjugate. Previously, we found that 6-ring-locked RAL with a ring structure that prevents isomerization around the $\mathrm{C}^{11}=\mathrm{C}^{12}$ double bond can specifically bind to rod opsin in vitro and in vivo. Rod cells were only marginally active with 11-cis-6-ring-retinal even 
when exposed to bright light (Jang et al., 2001; Kuksa et al., 2002). Green cone pigment does not regenerate with this chromophore (Alexander et al., 2017).

The 11-cis-6-ring-RAL-CM-chitosan conjugate (compound C1) was synthesized by a method similar to that used for the 9-cis-RAL-CM-chitosan conjugate. The scheme of synthetic reactions is shown in Fig. 7A. CM-chitosan reacted with 11-cis6-ring-RAL in MES buffer, $\mathrm{pH}$ 6.3, to produce the 11-cis-6ring-RAL-CM-chitosan conjugate (compound $\mathrm{C} 1$ ) that is a Schiff-base. The imine group in the Schiff-base compound C1 can be reduced by reaction with $\mathrm{NaBH}_{4}$ to form a new CM-chitosan conjugate, compound C2. Both these 11-cis-6ring-RAL-CM-chitosan conjugates were characterized by their UV spectra (Fig. 7B). The maximum absorbance of the 11-cis6-ring-RAL-CM-chitosan conjugate (compound C1) was $363 \mathrm{~nm}$. Reduction of the Schiff-base led to a blue-shift of $46 \mathrm{~nm}$ documented by UV-Vis spectroscopy of the maximum absorbance of the CM-chitosan conjugate (compound $\mathrm{C} 2, \lambda=$ $317 \mathrm{~nm})$ compared with compound C1 $(\lambda=363 \mathrm{~nm})$. Moreover, a red-shift of 11-cis-6-ring-RAL-CM-chitosan conjugate (compound $\mathrm{C} 1, \lambda=363 \mathrm{~nm}$ ) was observed compared with 9-cisRAL-CM-chitosan conjugate $(\lambda=350 \mathrm{~nm})$. We attribute this finding to the red-shift of the 11-cis-6-ring-RAL monomer $(\lambda=$ $374 \mathrm{~nm})$ relative to the 9 -cis-RAL monomer $(\lambda=365 \mathrm{~nm})$.

Regeneration of Visual Pigment with Both 9-cis-RAL and 11-cis-6-Ring-RAL Chromophore Analogs In Vivo and In Vitro. Visual pigment was regenerated with 9-cisRAL or 11-cis-6-ring-RAL chromophore analogs in vivo in $\mathrm{Lrat}^{-1-}$ mice lacking production of the endogenous chromophore 11-cis-RAL and in vitro in opsin-containing ROS membranes isolated from bovine retinas. To determine pigment regeneration in vivo, eyes from treated and untreated $\mathrm{Lrat}^{-1-}$ mice were collected and pigments were purified by 1D4 immunoaffinity chromatography. In parallel, pigments were regenerated with chromophore analogs in bovine ROS membranes. UV-Vis spectra obtained from the purified proteins indicated formation of iso-Rho. In both proteins purified from mouse eyes and bovine ROS, the appearance of an absorption maximum peak at $485 \mathrm{~nm}$ after regeneration with 9-cis-RAL and an absorption maximum peak at $505 \mathrm{~nm}$ after regeneration with 11-cis-6-ring-RAL were detected, whereas no comparable absorption maximum peaks were observed in protein samples purified from untreated mice and untreated bovine ROS (Fig. 8).
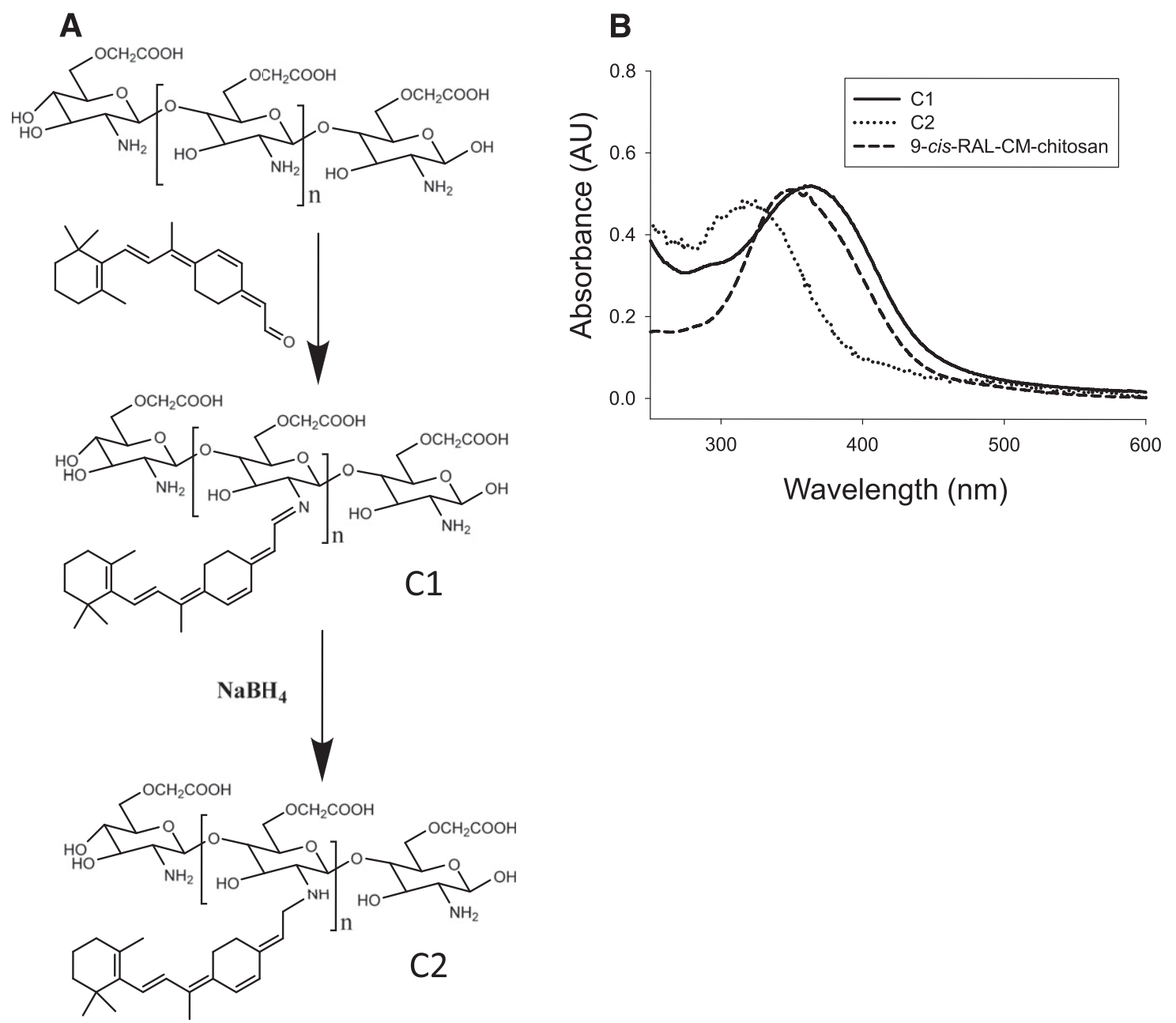

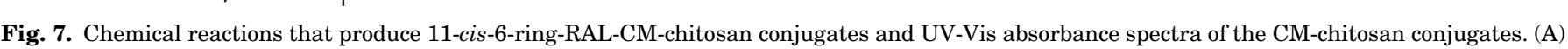

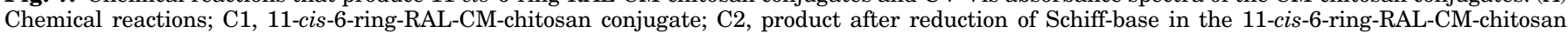
conjugate. (B) UV-Vis absorbance spectra of the CM-chitosan conjugate unreduced form (C1) and reduced form (C2). 
A

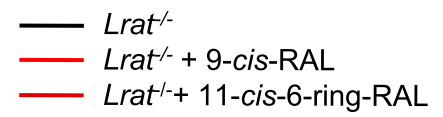

B
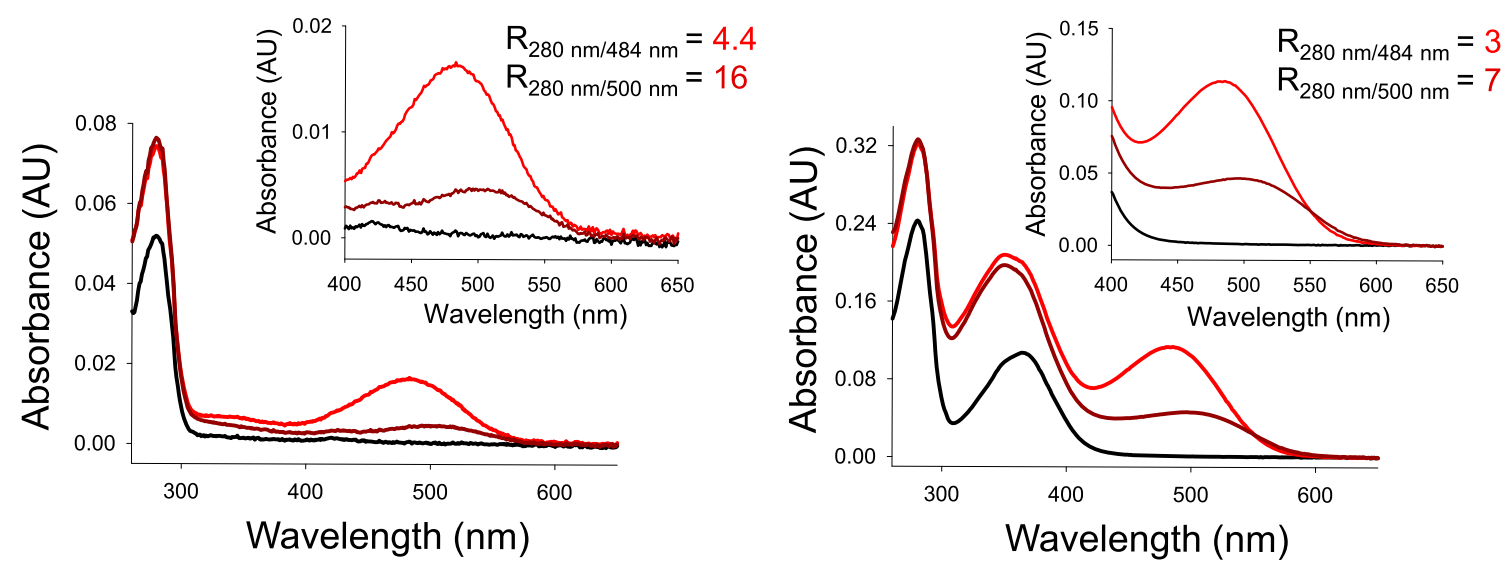

Fig. 8. Regeneration of opsin with 9-cis-RAL administered together with 11-cis-6-ring-RAL. Iso-Rho was regenerated with 9-cis-RAL and 11-cis-6-ringRAL, both in $\mathrm{Lrat}^{-1-}$ mice in vivo and in opsin-containing ROS membranes isolated from bovine retinas. (A) $\mathrm{Lrat}^{-1-}$ mice treated with either 9-cis-RAL or 11-cis-6-ring-RAL. After treatment, eyes from three animals were used for pigment purification by 1D4 affinity chromatography. UV-Vis absorption spectrum measured in the sample from $\mathrm{Lrat}^{-1-}$ mice treated with 9-cis-RAL (red spectrum) indicates an absorption maximum at $485 \mathrm{~nm}$, whereas an absorption maximum at $505 \mathrm{~nm}$ was detected in the protein sample purified from $\mathrm{Lrat}^{-1}$ mice treated with 11-cis-6-ring-RAL (dark red spectrum). Opsin purified from untreated $\mathrm{Lrat}^{-1-}$ mice had no absorbance peak at $\sim 500 \mathrm{~nm}$ (black spectrum). (B) UV-Vis absorption spectra of opsin membranes regenerated with 9-cis-RAL or 11-cis-6-ring-RAL showed absorbance peaks at $485 \mathrm{~nm}$ (red spectrum) and $505 \mathrm{~nm}$ (dark red spectrum), respectively. Unregenerated opsin membranes had no absorbance peak at $\sim 500 \mathrm{~nm}$ (black spectrum). The peak at $\sim 360 \mathrm{~nm}$ indicates the presence of residual all-transRAL released from the chromophore-binding pocket during opsin membrane preparation (see Materials and Methods).

11-cis-6-Ring-RAL-CM-Chitosan Conjugate and 9-cisRAL-CM-Chitosan Conjugate Improve RAL Function in Lrat $^{-1-}$ Mice. To compare 11-cis-6-ring-RAL and its chitosan conjugate with 9-cis-RAL and its conjugate in rescuing of visual function, 4-week-old $\mathrm{Lrat}^{-1-}$ mice were reared in the dark and divided into eight groups. For the $\mathrm{Lrat}^{-1-}$ control group, mice were gavaged with soybean oil or CM-chitosan. For the 11-cis-6-ring-RAL group, mice were gavaged with 11-cis-6-ring-RAL or 11-cis-6-ring-RAL-CMchitosan conjugate at a dose of $40 \mathrm{mg} / \mathrm{kg}$ of 11-cis-6-ring-RAL or its equivalent 48 hours prior to ERG evaluation. For the 9-cis-RAL group, mice were gavaged with 9-cis-RAL or 9-cisRAL-CM-chitosan conjugate at a dose of $40 \mathrm{mg} / \mathrm{kg}$ of 9 -cis-RAL or its equivalent 48 hours prior to ERG evaluation. For the 11-cis-6-ring-RAL plus 9-cis-RAL group, mice were gavaged with 11-cis-6-ring-RAL or 11-cis-6-ring-RAL-CM-chitosan conjugate at a dose of $40 \mathrm{mg} / \mathrm{kg}$ of 11-cis-6-ring-RAL or its equivalent at day 1 and day 3 , and 9-cis-RAL or 9-cis-RAL-CMchitosan conjugate at a dose of $40 \mathrm{mg} / \mathrm{kg}$ of 9 -cis-RAL or its equivalent at day 5, and the ERG evaluation was performed at day 7. ERG responses were recorded under both scotopic and photopic conditions, and amplitudes of the b-waves at $1.6 \mathrm{log} \mathrm{cd} \cdot \mathrm{s} \cdot \mathrm{m}^{-2}$ are summarized in Fig. 9A. For scotopic ERG b-waves, treatment with 9-cis-RAL or 11-cis-6-ring-RAL produced significant increases in amplitudes compared with the vehicle control group; treatment with conjugates produced significant increases in amplitude compared with the unconjugated drug treatment groups; treatment with 9-cis-RAL produced significant increases in amplitudes compared with the 11-cis-6-ring-RAL treatment group; treatment with 11-cis6-ring-RAL plus 9-cis-RAL produced significant increases in amplitudes compared with the 9-cis-RAL treatment group. UV-Vis absorption spectra of rod pigment purified from mice treated with 9-cis-RAL or 11-cis-6-ring-RAL indicated pigment regeneration with a peak of absorption at $485 \mathrm{~nm}$ for 9-cis-RAL regenerated iso-Rho and a smaller peak at $505 \mathrm{~nm}$ for 11-cis-6-ring-RAL regenerated pigment (Fig. 9B). Pigment isolated from mice treated with 9-cis-RAL and 11-cis-6-ringRAL had the highest peak of absorption among all four groups, in parallel with the highest scotopic ERG responses. Scotopic ERGs reflect the response primarily from rod cells, and their increase in amplitudes indicates restoration of rod pigment and improvement of rod cell function. For photopic ERG b-waves, treatment with 11-cis-6-ring-RAL or 9-cis-RAL produced significant increases in amplitudes compared with the vehicle-treatment control group. However, there was no significant difference in the amplitudes of the photopic ERG b-waves between all treatment groups. Because photopic ERGs reflect the response primarily from cone cells, similar amplitudes of photopic ERG b-waves indicate formation of cone pigment at similar levels, resulting in the similar improvement of cone cell function in all treatment groups. This suggests that 11-cis-6-ring-RAL does not affect cone pigment regeneration as published previously (Alexander et al., 2017), but may block rhodopsin photochemistry.

9-cis-RAL-CM-chitosan Conjugate Improves Photoreceptor Function in RPE65-deficient Dogs. The scotopic and photopic ERG responses of RPE65-deficient dogs prior to treatment were below detectable limits to most stimuli $(<1$ $\mu \mathrm{V}$ in amplitude), but in some dogs a very small response to stronger stimuli was noted (1-3.5 $\mu \mathrm{V}$ in amplitude, Supplemental Tables 1-6). Following intravitreal injection of 9-cisRAL-CM-chitosan, scotopic and photopic ERG responses were present, indicating improvement in both rod and cone responses (Fig. 10, A and B; Supplemental Tables 1-6; Table 1). There also appeared to be a dose effect (Fig. 10C). Table 1 

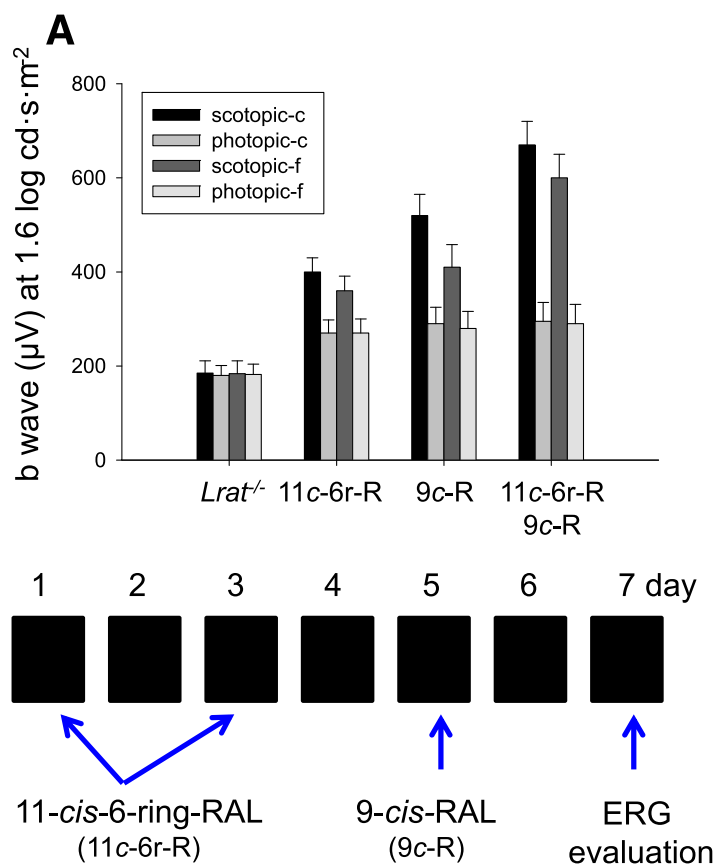

B
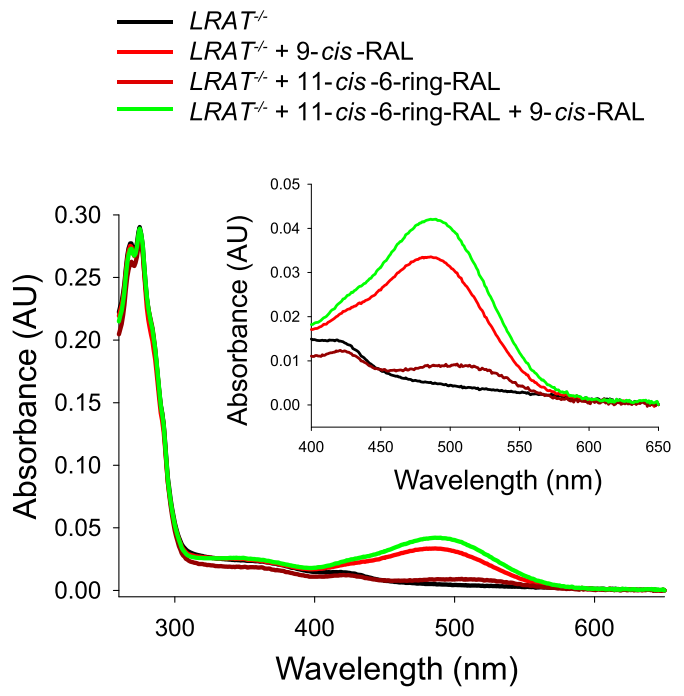

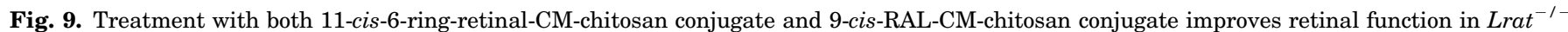

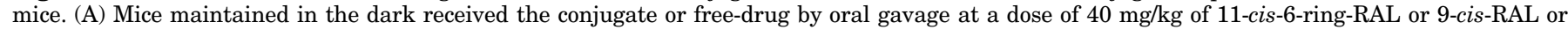

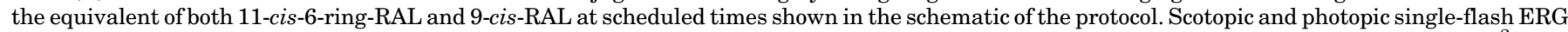

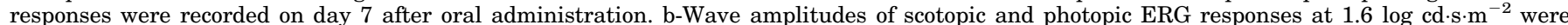

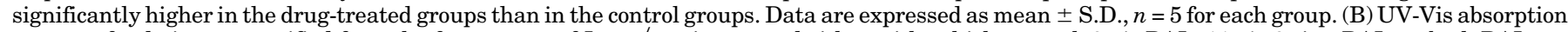

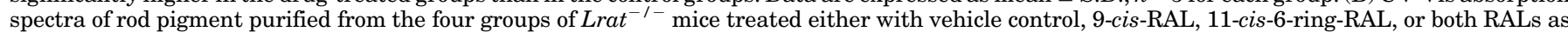

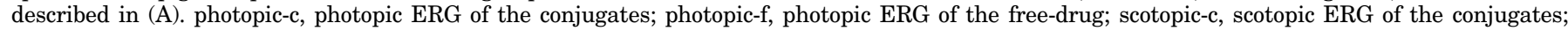

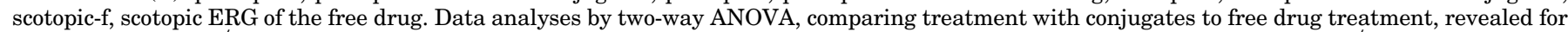

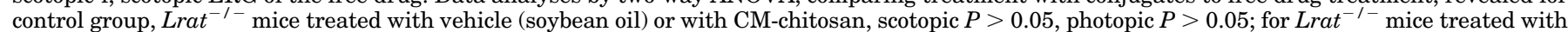

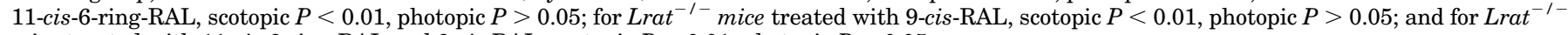
mice treated with 11-cis-6-ring-RAL and 9-cis-RAL, scotopic $P<0.01$, photopic $P>0.05$.

shows the scotopic ERG a- and b-wave amplitudes to stimuli of 3 and $10 \mathrm{~cd} \cdot \mathrm{s} \cdot \mathrm{m}^{-2}$ for RPE65-deficient dogs treated with intravitreal 9-cis-RAL-CM-chitosan conjugate with statistical comparison of the mean ERG amplitudes pre- and posttreatment. The peak recorded responses were at 10 days postinjection, after which there was a slow and progressive decline in amplitudes such that at 54 days postinjection the mean ERG amplitudes in the eyes injected with 9-cis-RAL-CM-chitosan at $100 \%$ concentration had returned almost to preinjection levels (Fig. 10D). There was a more rapid decline in the photopic ERG rescue and at lower doses (data not shown).

\section{Discussion}

Many retinal degenerative diseases are known to be associated with defects in the retinoid (visual) cycle (Travis et al., 2007). Thanks to painstaking biochemical reconstitution studies supported by genetically engineered animal models and genetic/phenotypic studies of humans with specific blinding diseases, a molecular understanding of the retinoid cycle in the mammalian retina has advanced appreciably over the past few years (Kiser et al., 2014). Understanding the fundamental biochemical processes underlying diseases that involve the retinoid cycle is then essential for the development of effective therapeutics. A successful clinical trial recently reported that pharmacological doses of 9-cisRAL could dramatically restore visual function in humans with genetic disease involving the retinoid cycle (Koenekoop et al., 2014). Because artificial illumination renders rod vision less critical than cone vision, we need to develop improved methods for sustained delivery of retinoids predominantly to improve the function and preserve the structure of cone photoreceptors.

We already have demonstrated that mechanism-based pharmacological interventions can restore vision in otherwise incurable genetic retinal dystrophies and further improvements are possible (Van Hooser et al., 2000, 2002; Batten et al., 2005). The current study centers on two straightforward findings: first, we found that conjugation of 9-cis-RAL with chitosan results in a sustained 9-cis-RAL release profile. This finding can be translated into improvements in retinoid-based therapeutic strategies. Second, we also found that ring-locked retinal that prevents isomerization around the $\mathrm{C}^{11}=\mathrm{C}^{12}$ double bond specifically binds and blocks rod opsin but not cone opsins, thereby permitting a specific reduction of chromophore consumption by rod cells and increasing retinoid availability for the regeneration of cone pigments. The simple chemistry and direct conjugation of retinoids to CM-chitosan could be a potentially outstanding way for sustained delivery of these compounds. Although this complicates the treatment and approval process, a major benefit could be lower doses of therapeutic retinoids, thereby minimizing their potential side effects. Although no toxicology profile is available for 11-cis-6ring-RAL, specifically how this compound could affect retinoid acid signaling when oxidized, our data support further work in this area of therapeutic intervention. With approaches in gene 
A

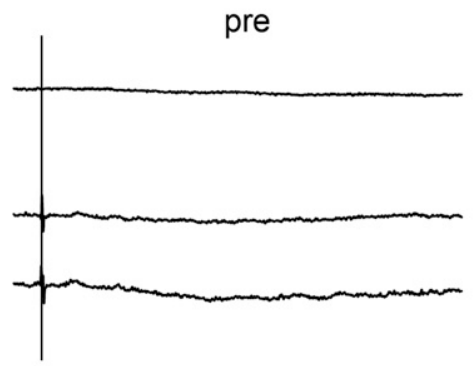

$\begin{array}{lllllll}0 & 50 & 100 & 150 \quad 200 \quad 250 \quad 300\end{array}$ Time (ms)

\section{B}
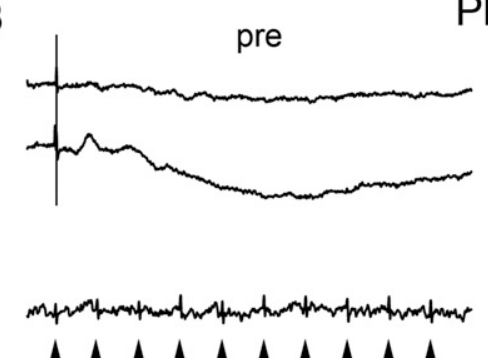

$\wedge \wedge \wedge \wedge \wedge \wedge \wedge \wedge \wedge$

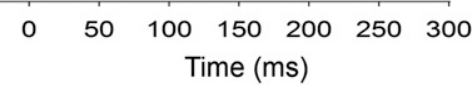

Scotopic

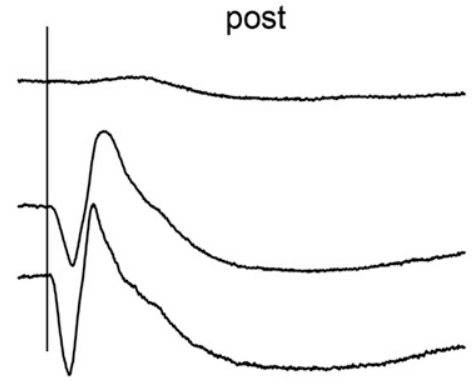

$\begin{array}{lllllll}0 & 50 & 100 & 150 & 200 & 250 & 300\end{array}$ Time (ms)

Photopic
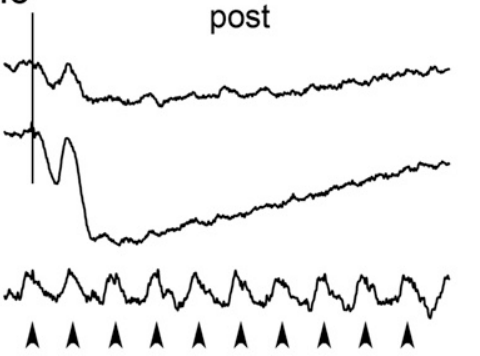

$\begin{array}{lllllll}0 & 50 \quad 100 & 150 & 200 & 250 & 300\end{array}$ Time (ms)

C

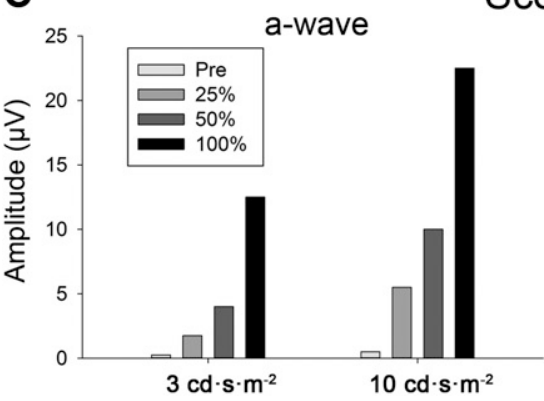

Scotopic

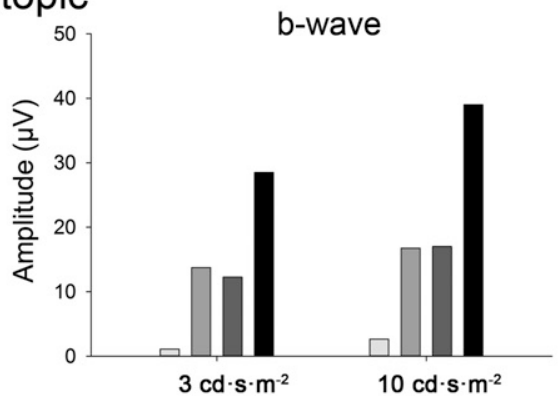

D

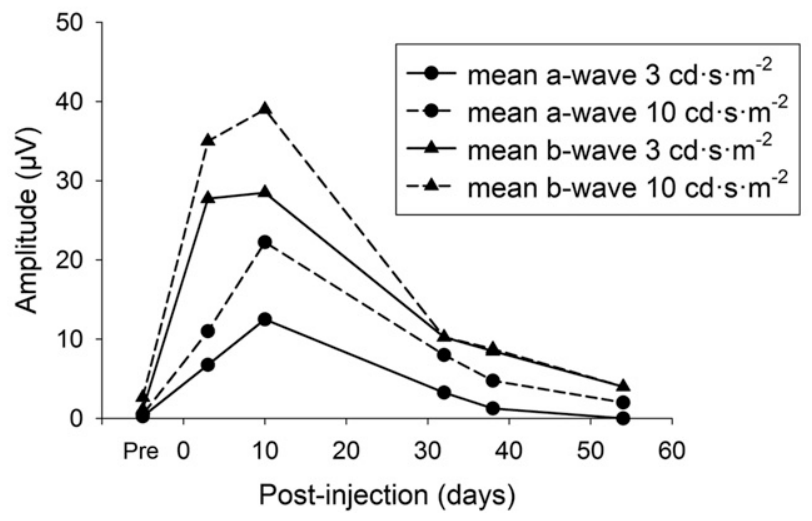

Fig. 10. Retinal function following intravitreal administration of 9-cis-RAL-CM-chitosan conjugate in Rpe65-deficient dogs. (A) Scotopic and (B) photopic ERG amplitudes before and 10 days after intravitreal 9-cis-RAL-CM-chitosan conjugate administration. Scotopic stimuli were $0.01,3$, and $10 \mathrm{~cd} \cdot \mathrm{s} \cdot \mathrm{m}^{-2}$. Photopic single flash stimuli, 3 and $10 \mathrm{~cd} \cdot \mathrm{s} \cdot \mathrm{m}^{-2}$ and $33 \mathrm{~Hz} 3 \mathrm{~cd} \cdot \mathrm{s} \cdot \mathrm{m}^{-2}$ flicker on $30 \mathrm{~cd} \cdot \mathrm{m}^{-2}$ background light. Size bars: scotopic $20 \mu \mathrm{V}$; photopic $10 \mu \mathrm{V}$. (C) Mean scotopic a- and b-wave amplitudes for eyes injected with 9-cis-RAL-CM-chitosan conjugate at full concentration and at $25 \%$ and $50 \%$ of full concentration 10 days following injection ( $n=2$ for each concentration). (D) Mean a- and b-wave scotopic amplitudes with time after injection of eyes treated with $100 \%$ 9-cis-RAL-CM-chitosan conjugate. Initial peak ERG amplitudes at 10 days postinjection revealed a decline toward preinjection amplitudes at 54 days postinjection. therapy recently approved by the Food and Drug Administration for LCA patients, chromophore supplementation therapy as described in this study could serve in the capacity as a combination treatment. One limitation, however, should be noted: retinoid therapy would not be recommended for pregnant women because it may interfere with visiatin $\mathrm{A}$ in the developing fetus (Mahan and Vallet, 1997; Chassaing et al., 2009).

Chitosan, obtained from chitin shells of shrimps by base hydrolysis, and its carboxymethylated derivative CM-chitosan are used as carriers for drug delivery. Both appear to be safe, 
because they are also used as part of antibacterial preparations, cosmetics, food preservatives, and included in bandages to reduce bleeding. Chitosan, composed of randomly distributed $\beta$-(1 $\rightarrow 4$ )-linked D-glucosamine and $N$-acetylated D-glucosamine, has little if any toxicity but is highly biodegradable (Paolicelli et al., 2009; Upadhyaya et al., 2014; Hu et al., 2017; Kalliola et al., 2017). The free amino group of chitosan has a pKa of about 6.5, making it ideal for Schiff base conjugation of functional aldehydes (like RAL). Chitosan, modified by carboxymethylation, displays a marked increase in solubility at neutral and alkaline $\mathrm{pH}$ without adversely affecting its other properties. However, it has yet to be approved by the Food and Drug Administration as a vehicle for either drug or nonviral gene delivery.

The water solubility of a 9-cis-RAL-CM-chitosan conjugate depends on its content of 9-cis-retinoid. Because CM-chitosan is a water-soluble polysaccharide, whereas 9-cis-retinoid is hydrophobic, the higher the content of 9-cis-retinoid in the CM-chitosan conjugate the lower its water solubility. The 9-cisRAL-CM-chitosan conjugate is produced by attaching 9-cis-RAL to CM-chitosan via a covalent bond. The higher 9-cis-retinoid content means that more 9-cis-retinoid is attached to a given amount of CM-chitosan, making the CM-chitosan conjugate more hydrophobic. Also, hydrophobic portions of the CM-chitosan conjugate can aggregate and thus lower aqueous solubility. Moreover, water-insoluble CM-chitosan conjugates can block cleavage of the covalent bond that connects the drug to CM-chitosan and thereby hinder drug release from the CM-chitosan conjugate, allowing for a more sustained drug delivery.

To demonstrate the ability of the 9-cis-RAL-CM-chitosan conjugate to supply 9-cis-RAL and improve photoreceptor function in a large animal model, the RPE65-deficient dog was chosen. Our previous studies with this model, which lacks visual cycle function, showed that direct intravitreal administration of 9-cis-RAL temporarily improved retinal function (Gearhart et al., 2010). The dog, and other carnivores, differ from other mammals in their circulatory vitamin A transport (Raila et al., 2002). In this respect, vitamin A metabolism in mice more closely resembles the conditions in humans (Blaner et al., 2016). A pilot study of oral administration of the 9-cisRAL-CM-chitosan conjugate failed to rescue photoreceptor function in the RPE65-deficient dog, most likely because of the vitamin A transport peculiarities in this species. Therefore, to test the bioavailability of 9-cis-RAL from the conjugate, intravitreal administration was used. This resulted in rescue of rod and cone photoreceptor function, further confirming that 9-cis-RAL was available to the photoreceptors. The ERG amplitudes were markedly improved over those of untreated RPE65-deficient dogs, and achieved $\sim 25 \%$ of wild-type levels in response to the International Society for Clinical Electrophysiology of Vision standard flash stimulus. Our previous study using unconjugated intravitreal 9-cis-RAL had resulted in a similar degree of ERG rescue (Gearhart et al., 2010).

In summary, defects in most retinoid cycle genes can cause retinal degeneration in humans, ranging from childhood diseases such as LCA to late-onset disease. Both gene therapy and pharmacological approaches have been translated into the clinic where promising first-in-human data were obtained, but limitations of these therapies also were noted (Zhang et al., 2015). Due to better understanding of the retinoid cycle, improved interventions are now feasible. 
For example, the interplay between rod and cone photoreceptor regeneration described here promises to make it possible to deliver smaller amounts of active chromophore exclusively to cone cells, while blocking rod bleaching and regeneration cycles during day-time vision. Moreover, the higher levels of active agent following the delivery of the unconjugated drug can cause toxic side effects, but this problem can be alleviated by the slower delivery of chromophore through the digestive tract using simple conjugated prodrugs. As demonstrated in two animal models deficient in the production of active chromophore, administration of conjugated 9-cis-RAL is an effective approach to improve retinal function, and when it is possible to deliver it in a sustained manner via the GI tract has advantageous properties over simple dosing with free retinoid.

\section{Acknowledgments}

The authors thank Dr. Leslie T. Webster and the members of the Palczewski laboratory for valuable comments regarding this manuscript. We are also grateful to Dr. Muneto Mogi (Novartis Inc.) for 11-cis-6-ring-RAL. We thank Heather Defore for assistance with the dog studies.

\section{Authorship Contributions}

Participated in research design: Gao, Kahremany, Zhang, Jastrzebska, Querubin, Petersen-Jones, Palczewski.

Conducted experiments: Gao, Kahremany, Zhang, Jastrzebska, Querubin, Petersen-Jones.

Contributed new reagents or analytical tools: Kahremany.

Performed data analysis: Gao, Kahremany, Zhang, Jastrzebska, Querubin, Petersen-Jones, Palczewski.

Wrote or contributed to writing of the manuscript: Gao, Kahremany, Zhang, Jastrzebska, Petersen-Jones, Palczewski.

\section{References}

Alexander NS, Katayama K, Sun W, Salom D, Gulati S, Zhang J, Mogi M, Palczewski $\mathrm{K}$, and Jastrzebska B (2017) Complex binding pathways determine the regeneration of mammalian green cone opsin with a locked retinal analogue. J Biol Chem 292:10983-10997.

Annear MJ, Bartoe JT, Barker SE, Smith AJ, Curran PG, Bainbridge JW, Ali RR, and Petersen-Jones SM (2011) Gene therapy in the second eye of RPE65-deficient dogs improves retinal function. Gene Ther 18:53-61.

Bainbridge JW, Smith AJ, Barker SS, Robbie S, Henderson R, Balaggan K, Viswanathan A, Holder GE, Stockman A, Tyler N, et al. (2008) Effect of gene therapy on visual function in Leber's congenital amaurosis. $N$ Engl J Med 358:2231-2239.

Batten ML, Imanishi Y, Maeda T, Tu DC, Moise AR, Bronson D, Possin D, Van Gelder RN, Baehr W, and Palczewski K (2004) Lecithin-retinol acyltransferase is essential for accumulation of all-trans-retinyl esters in the eye and in the liver. $J$ Biol Chem 279:10422-10432.

Batten ML, Imanishi Y, Tu DC, Doan T, Zhu L, Pang J, Glushakova L, Moise AR, Baehr W, Van Gelder RN, et al. (2005) Pharmacological and rAAV gene therapy rescue of visual functions in a blind mouse model of Leber congenital amaurosis. PLoS Med 2:e333.

Bhattacharya S, Ridge KD, Knox BE, and Khorana HG (1992) Light-stable rhodopsin. I. A rhodopsin analog reconstituted with a nonisomerizable 11-cis retinal derivative. J Biol Chem 267:6763-6769

Blaner WS, Li Y, Brun PJ, Yuen JJ, Lee SA, and Clugston RD (2016) Vitamin A absorption, storage and mobilization. Subcell Biochem 81:95-125.

Brown PK and Wald G (1956) The neo-b isomer of vitamin A and retinene. $J$ Biol Chem 222:865-877.

Chassaing N, Golzio C, Odent S, Lequeux L, Vigouroux A, Martinovic-Bouriel J, Tiziano FD, Masini L, Piro F, Maragliano G, et al. (2009) Phenotypic spectrum of STRA6 mutations: from Matthew-Wood syndrome to non-lethal anophthalmia. Hum Mutat 30:E673-E681.

Cideciyan AV, Aleman TS, Boye SL, Schwartz SB, Kaushal S, Roman AJ, Pang JJ, Sumaroka A, Windsor EA, Wilson JM, et al. (2008) Human gene therapy for RPE65 isomerase deficiency activates the retinoid cycle of vision but with slow rod kinetics. Proc Natl Acad Sci USA 105:15112-15117.

Cideciyan AV, Jacobson SG, Beltran WA, Sumaroka A, Swider M, Iwabe S, Roman AJ, Olivares MB, Schwartz SB, Komáromy AM, et al. (2013) Human retinal gene therapy for Leber congenital amaurosis shows advancing retinal degeneration despite enduring visual improvement. Proc Natl Acad Sci USA 110:E517-E525.

Gearhart PM, Gearhart C, Thompson DA, and Petersen-Jones SM (2010) Improvement of visual performance with intravitreal administration of 9-cis-retinal in Rpe65-mutant dogs. Arch Ophthalmol 128:1442-1448.

Gulati S, Jastrzebska B, Banerjee S, Placeres AL, Miszta P, Gao S, Gunderson K Tochtrop GP, Filipek S, Katayama K, et al. (2017) Photocyclic behavior of rhodopsin induced by an atypical isomerization mechanism. Proc Natl Acad Sci USA 114:E2608-E2615.

Hu L, Zhang P, Wang X, Cheng X, Qin J, and Tang R (2017) pH-sensitive carboxymethyl chitosan hydrogels via acid-labile ortho ester linkage for potential biomedical applications. Carbohydr Polym 178:166-179.

Jang GF, Kuksa V, Filipek S, Bartl F, Ritter E, Gelb MH, Hofmann KP, and Palczewski K (2001) Mechanism of rhodopsin activation as examined with ring-constrained retinal analogs and the crystal structure of the ground state protein. J Biol Chem 276:26148-26153.

Jastrzebska B, Golczak M, Fotiadis D, Engel A, and Palczewski K (2009) Isolation and functional characterization of a stable complex between photoactivated rhodopsin and the G protein, transducin. FASEB $J$ 23:371-381.

Jastrzebska B, Orban T, Golczak M, Engel A, and Palczewski K (2013) Asymmetry of the rhodopsin dimer in complex with transducin. FASEB $J$ 27:1572-1584.

Kalliola S, Repo E, Srivastava V, Heiskanen JP, Sirviö JA, Liimatainen H, and Sillanpää M (2017) The $\mathrm{pH}$ sensitive properties of carboxymethyl chitosan nanoparticles cross-linked with calcium ions. Colloids Surf B Biointerfaces 153: 229-236.

Kiser PD, Golczak M, Maeda A, and Palczewski K (2012) Key enzymes of the retinoid (visual) cycle in vertebrate retina. Biochim Biophys Acta 1821:137-151.

Kiser PD, Golczak M, and Palczewski K (2014) Chemistry of the retinoid (visual) cycle. Chem Rev 114:194-232.

Kiser PD and Palczewski K (2016) Retinoids and retinal diseases. Annu Rev Vis Sci 2 : 197-234

Koenekoop RK, Sui R, Sallum J, van den Born LI, Ajlan R, Khan A, den Hollander AI, Cremers FP, Mendola JD, Bittner AK, et al. (2014) Oral 9-cis retinoid for childhood blindness due to Leber congenital amaurosis caused by RPE65 or LRAT mutations: an open-label phase $1 \mathrm{~b}$ trial. Lancet 384:1513-1520.

Kuksa V, Bartl F, Maeda T, Jang GF, Ritter E, Heck M, Preston Van Hooser J, Liang Y, Filipek S, Gelb MH, et al. (2002) Biochemical and physiological properties of rhodopsin regenerated with 11-cis-6-ring- and 7-ring-retinals. J Biol Chem $\mathbf{2 7 7}$ $42315-42324$.

Lamb TD and Pugh EN, Jr (2004) Dark adaptation and the retinoid cycle of vision. Prog Retin Eye Res 23:307-380

Maeda T, Cideciyan AV, Maeda A, Golczak M, Aleman TS, Jacobson SG, and Palczewski K (2009a) Loss of cone photoreceptors caused by chromophore depletion is partially prevented by the artificial chromophore pro-drug, 9-cisretinyl acetate. Hum Mol Genet 18:2277-2287.

Maeda T, Dong Z, Jin H, Sawada O, Gao S, Utkhede D, Monk W, Palczewska G, and Palczewski K (2013) QLT091001, a 9-cis-retinal analog, is well-tolerated by retinas of mice with impaired visual cycles. Invest Ophthalmol Vis Sci 54:455-466.

Maeda A, Golczak M, Chen Y, Okano K, Kohno H, Shiose S, Ishikawa K, Harte W, Palczewska G, Maeda T, et al. (2011) Primary amines protect against retinal degeneration in mouse models of retinopathies. Nat Chem Biol 8:170-178.

Maeda T, Maeda A, Leahy P, Saperstein DA, and Palczewski K (2009b) Effects of long-term administration of 9-cis-retinyl acetate on visual function in mice. Invest Ophthalmol Vis Sci 50:322-333.

Maeda A, Maeda T, and Palczewski K (2006) Improvement in rod and cone function in mouse model of Fundus albipunctatus after pharmacologic treatment with 9-cisretinal. Invest Ophthalmol Vis Sci 47:4540-4546.

Maguire AM, High KA, Auricchio A, Wright JF, Pierce EA, Testa F, Mingozzi F, Bennicelli JL, Ying GS, Rossi S, et al. (2009) Age-dependent effects of RPE65 gene therapy for Leber's congenital amaurosis: a phase 1 dose-escalation trial. Lancet 374:1597-1605.

Mahan DC and Vallet JL (1997) Vitamin and mineral transfer during fetal development and the early postnatal period in pigs. J Anim Sci 75:2731-2738.

Mata NL, Radu RA, Clemmons RC, and Travis GH (2002) Isomerization and oxidation of vitamin a in cone-dominant retinas: a novel pathway for visual-pigment regeneration in daylight. Neuron 36:69-80.

McCulloch DL, Marmor MF, Brigell MG, Hamilton R, Holder GE, Tzekov R, and Bach M (2015) ISCEV Standard for full-field clinical electroretinography (2015 update). Doc Ophthalmol 130:1-12

McKibbin C, Farmer NA, Jeans C, Reeves PJ, Khorana HG, Wallace BA, Edwards PC, Villa C, and Booth PJ (2007) Opsin stability and folding: modulation by phospholipid bicelles. J Mol Biol 374:1319-1332.

Palczewski K, Van Hooser JP, Garwin GG, Chen J, Liou GI, and Saari JC (1999) Kinetics of visual pigment regeneration in excised mouse eyes and in mice with a targeted disruption of the gene encoding interphotoreceptor retinoid-binding protein or arrestin. Biochemistry 38:12012-12019.

Paolicelli P, de la Fuente M, Sánchez A, Seijo B, and Alonso MJ (2009) Chitosan nanoparticles for drug delivery to the eye. Expert Opin Drug Deliv 6:239-253.

Papermaster DS (1982) Preparation of retinal rod outer segments. Methods Enzymol 81:48-52.

Raila J, Radon R, Trüpschuch A, and Schweigert FJ (2002) Retinol and retinyl ester responses in the blood plasma and urine of dogs after a single oral dose of vitamin A. J Nutr 132(6 Suppl 2)1673S-1675S

Robeson CD, Blum WP, Dieterle JM, Cawley JD, and Baxter JG (1955) Chemistry of vitamin-A. 25. Geometrical isomers of vitamin-a aldehyde and an isomer of its alpha-ionone analog. J Am Chem Soc 77:4120-4125.

Scholl HP, Moore AT, Koenekoop RK, Wen Y, Fishman GA, van den Born LI, Bittner A, Bowles K, Fletcher EC, Collison FT, et al.; RET IRD 01 Study Group (2015) Safety and proof-of-concept study of oral QLT091001 in retinitis pigmentosa due to inherited deficiencies of retinal pigment epithelial 65 protein (RPE65) or Lecithin: Retinol Acyltransferase (LRAT). PLoS One 10:e0143846.

Spalink JD, Reynolds AH, Rentzepis PM, Sperling W, and Applebury ML (1983) Bathorhodopsin intermediates from 11-cis-rhodopsin and 9-cis-rhodopsin. Proc Natl Acad Sci USA 80:1887-1891.

Sundermeier TR, Zhang N, Vinberg F, Mustafi D, Kohno H, Golczak M, Bai X, Maeda A, Kefalov VJ, and Palczewski K (2014) DICER1 is essential for survival of postmitotic rod photoreceptor cells in mice. FASEB $J$ 28:3780-3791. 
Surya A, Foster KW, and Knox BE (1995) Transducin activation by the bovine opsin apoprotein. J Biol Chem 270:5024-5031.

Tian H, Sakmar TP, and Huber T (2017) The energetics of chromophore binding in the visual photoreceptor rhodopsin. Biophys $J$ 113:60-72.

Travis GH, Golczak M, Moise AR, and Palczewski K (2007) Diseases caused by defects in the visual cycle: retinoids as potential therapeutic agents. Annu Rev Pharmacol Toxicol 47:469-512.

Upadhyaya L, Singh J, Agarwal V, and Tewari RP (2014) The implications of recent advances in carboxymethyl chitosan based targeted drug delivery and tissue engineering applications. $J$ Control Release 186:54-87.

Van Hooser JP, Aleman TS, He YG, Cideciyan AV, Kuksa V, Pittler SJ, Stone EM, Jacobson SG, and Palczewski K (2000) Rapid restoration of visual pigment and function with oral retinoid in a mouse model of childhood blindness. Proc Natl Acad Sci USA 97:8623-8628.

Van Hooser JP, Liang Y, Maeda T, Kuksa V, Jang GF, He YG, Rieke F, Fong HK Detwiler PB, and Palczewski K (2002) Recovery of visual functions in a mouse model of Leber congenital amaurosis. J Biol Chem 277:19173-19182. von Lintig J, Kiser PD, Golczak M, and Palczewski K (2010) The biochemical and structural basis for trans-to-cis isomerization of retinoids in the chemistry of vision. Trends Biochem Sci 35:400-410.

Wald G and Brown PK (1953) The molar extinction of rhodopsin. J Gen Physiol 37: 189-200.

Wang JS and Kefalov VJ (2009) An alternative pathway mediates the mouse and human cone visual cycle. Curr Biol 19:1665-1669.

Zhang N, Tsybovsky Y, Kolesnikov AV, Rozanowska M, Swider M, Schwartz SB, Stone EM, Palczewska G, Maeda A, Kefalov VJ, et al. (2015) Protein misfolding and the pathogenesis of ABCA4-associated retinal degenerations. Hum Mol Genet 24:3220-3237.

Address correspondence to: Krzysztof Palczewski, Department of Pharmacology, School of Medicine, Case Western Reserve University, 10900 Euclid Ave., Cleveland, OH 44106. E-mail: kxp65@case.edu 